\title{
Robust non-quadratic static output feedback controller design for Takagi-Sugeno systems using descriptor redundancy
}

\author{
Tahar Bouarar $^{1}$, Kevin Guelton ${ }^{2, *}$, Noureddine Manamanni ${ }^{2}$ \\ ${ }^{1}$ IRSEEM EA4353, ESIGELEC, Technopôle du Madrillet \\ Avenue Galilée - BP 10024, 76801 Saint-Etienne du Rouvray Cedex. \\ ${ }^{2}$ CReSTIC EA3804, Université de Reims Champagne Ardennes \\ Moulin de la Housse BP 1039, 51687 Reims Cedex 2, France. \\ *Corresponding author: kevin.guelton@univ-reims.fr, Tel. +33 3269132 61, Fax. +33 326913104
}

\begin{abstract}
This work concerns robust static output feedback controller (SOFC) design for uncertain and disturbed Takagi-Sugeno (TS) systems using an H-infinity criterion. The main result is based on a descriptor formulation of the closed-loop dynamics. The proposed approach allows avoiding appearance of crossing terms between the controller's and the TS system's input matrices leading to easier LMI formulation than existing studies in the literature. Moreover, the proposed SOFC design conditions don't require any restrictions on the output equation and allow dealing with unmeasurable premise variables. Indeed, taking advantage of the uncertain TS modeling, nonlinearities associated to unmeasurable premises variables can be reported from the nominal part to the uncertainties. To provide LMIs of less conservatism, the results are conducted in the non-quadratic framework. Finally, two numerical examples and a benchmark of a crane system are proposed to illustrate the efficiency of the SOFC design methodology.
\end{abstract}

Keywords: Takagi-Sugeno models, Robust Static Output Feedback Controller, Descriptor Redundancy, Non-Quadratic Stabilization, LMI, Hœ criterion, Unmeasurable premise variables. 


\section{Introduction}

Takagi-Sugeno fuzzy systems (Takagi and Sugeno, 1985) have shown their interests since they allow extending some of the linear control concepts to the nonlinear cases (Takagi and Wang, 2001). Indeed, a TS fuzzy model is a collection of linear time invariant systems blended together with nonlinear membership functions. Therefore, convenient control approaches for such systems have been proposed through the concept of Parallel Distributed Compensation (PDC) (Takagi and Wang, 2001; Sala et al., 2005). PDC controllers design has been studied using a quadratic Lyapunov functions, see (Takagi and Wang, 2001; Takagi et al., 1996; Sala et al., 2005) and references therein. These approaches remain conservative since they need to find common Lyapunov matrices for a set of linear matrix inequalities (LMI) constraints. Thus, many ways have been proposed to relax these conditions. For instance, relaxation schemes have been developed based on rewriting the closed-loop interconnection structure of the considered control plant (Xiaodong and Qingling, 2003; Tuan et al., 2001). Other works have considered piecewise Lyapunov functions (Johansson et al., 1999) and, more accurately with the fuzzy aggregation of TS models, through a non-quadratic approach (also called fuzzy Lyapunov approach) (Tanaka et al., 2003; Guerra and Vermeiren, 2004; Rhee and Won, 2006; Feng, 2006; Lam and Leung, 2007; Chang and Yang, 2010; Guerra et al., 2012). For a review of relaxation issues, one can refer to (Sala, 2009).

Among control theory, regarding to output stabilization of TS fuzzy models, many works have been done for observer-based controller (OBC) design (Tanaka et al., 1998; Ma et al., 1998; Yoneyama et al., 2000; Yoneyama et al., 2001; Guerra et al., 2006; Mansouri et al., 2009), dynamic output feedback controller (DOFC) design in both the quadratic and the non-quadratic case (Li et al., 2000; Assawinchaichote et al., 2004; Nguang and Shi, 2006; Zerar et al., 2008; Guelton et al., 2008; Guelton et al., 2009). Static Output Feedback Controllers (SOFC) are of some interests for practical applications since they only require available signals from the plant to be controlled and doesn't need any online differential equation solving, so reducing online computational cost (Syrmos et al., 1997). In (Huang 
and Nguang, 2006) and (Huang and Nguang, 2007), SOFC design methodologies for TS fuzzy systems are proposed in terms of Bilinear Matrix Inequalities instead of LMI. In (Chadli et al., 2002), LMI conditions have been proposed. However, in these studies, some restrictive modeling assumptions have been made such that the output equation must be linear (full column rank matrix) and without direct transfers from the inputs to the outputs. Note that these restrictions are due to the occurrence of crossing terms in the closed-loop formulation (Zerar et al., 2008; Syrmos et al., 1997; Zhou and Doyle, 1996). Moreover, the results proposed in these previous studies being quadratic, they may suffer from conservatism. Note also that, unmeasurable premises constitute an important problem in static output feedback since it is somewhat difficult to estimate unmeasured variables without introducing a dynamical equation (observer) in the feedback and so reduce the applicative interest of SOFC. This problem has been seldom treated in the literature dealing with SOFC for TS systems and has been the topic of a very recent work where premises selection in SOFC fuzzy structure has been proposed (Tognetti et al., 2012).

In (Tanaka et al., 2007), a descriptor redundancy formulation has been employed to derive new nonquadratic LMI stability conditions for state feedback PDC controllers. One of the interests of such approach is that it allows decoupling crossing terms in the closed-loop dynamics formulation (Bouarar et al., 2010). Therefore, based on this property, LMI based non-quadratic robust DOFC design has been proposed in (Guelton et al., 2008; Guelton et al., 2009). Following this way, one of our preliminary studies has dealt with SOF controller design (Bouarar et al., 2009). In the latter, in aid of the redundancy property, non-quadratic LMI based SOFC design using a fuzzy Lyapunov approach for nominal TS systems has been proposed. However, this preliminary result isn't relevant in the case of unmeasurable premises. Note that the above described studies dealing with continuous time TS models, complementary works has been recently done in the discrete time framework (which is reputed more favorable than the continuous time case for LMI purposes). Indeed, the descriptor redundancy has been recently used for OBC or SOFC design for discrete-time fuzzy models (Chang and Yang, 2011; Chadli 
and Guerra, 2012).

The goal of this paper is to propose a descriptor redundancy approach leading to strict LMI based robust non-quadratic SOFC design for the class of continuous time uncertain and disturbed TS systems without any restrictions on the output equations. Taking advantage of the uncertain plant, it will be emphasis through an example how this modeling approach is relevant to deals with unmeasured premise variables for SOFC design. Moreover, the non-quadratic framework will be considered with recent conservatism improvements (Mozelli et al., 2009b).

This paper is organized as follows. First, convenient notations and lemma will be described. Then, in section 3, the problem statement of robust SOFC design for TS fuzzy models is presented with highlights on the descriptor redundancy approach and unmeasurable premises. Afterward, relaxed nonquadratic LMI based robust SOFC design conditions are proposed for the considered class of uncertain TS fuzzy models. Then, an extension to uncertain TS models subject to external disturbances is proposed by the use of a $H_{\infty}$ criterion. Finally, two numerical examples are provided to show the effectiveness of the proposed approach and an engineering example of a crane system is proposed as a benchmark in simulation.

\section{Notations and Lemma}

In the sequel, when there is no ambiguity, the time $t$ in a time varying variable will be omitted for space convenience. As usual, in a matrix, $(*)$ indicates a symmetrical transpose quantity. Moreover $I$ denotes an identity matrix with appropriate dimension. Let us consider $h_{i}(z)$ as scalar convex functions, the matrices $Y_{i}$ and $T_{i j}$ for, $i \in\{1, \ldots, r\}$ and $j \in\{1, \ldots, l\}$, with appropriate dimensions, we will denote $Y_{h}=\sum_{i=1}^{r} h_{i}(z) Y_{i}, \quad T_{h h}=\sum_{k=1}^{r} \sum_{i=1}^{r} h_{k}(z) h_{i}(z) T_{i k} . \quad$ Finally, one denotes $\quad \dot{X}_{h}=\frac{d\left(\sum_{i=1}^{r} h_{i}(z) X_{i}\right)}{d t} \quad$ and 
$\stackrel{\cdot \cdot\left(X_{h}\right)^{-1}}{=}=\frac{d\left(\left(\sum_{i=1}^{r} h_{i}(z) X_{i}\right)^{-1}\right)}{d t}$.

The following lemmas will be useful in the following sections to lead to and to relax LMI conditions.

Lemma 1 (Zhou and Khargonekar, 1988):

For real matrices $X$ and $Y$ with appropriate dimensions and a positive scalar $\varepsilon$, we have:

$X^{T} Y+Y^{T} X \leq \varepsilon X^{T} X+\varepsilon^{-1} Y^{T} Y$

Lemma 2 (Tuan et al., 2001): Consider the Parameterized Matrix Inequality (PMI) given by:

$\sum_{k=1}^{r} \sum_{i=1}^{r} h_{k}(z) h_{i}(z) \Omega_{i k}<0$

with $h_{i}(z) \geq 0$ and $\sum_{i=1}^{r} h_{i}(z)=1$.

The PMI (2) is verified if the matrix inequalities (3) and (4) hold:

$\Omega_{i i}<0$, for all $i, j=1,2, \ldots, r$

$\frac{1}{r-1} \Omega_{i i}+\frac{1}{2}\left(\Omega_{i j}+\Omega_{j i}\right)<0$, for $1 \leq i \neq j \leq r$,

Note that there exist numerous relaxation schemes in the literature which can be used without affecting 
the mathematical development of the results proposed below, for a review on these techniques, see (Sala, 2009). Nevertheless, the one presented in lemma 2 (Tuan et al., 2001) constitutes a good compromise between conservatism and computational cost since it doesn't require additional slack variables.

\section{Problem statement}

Let us consider the class of uncertain and disturbed TS fuzzy systems described by:

$$
\left\{\begin{array}{l}
\dot{x}(t)=\sum_{i=1}^{r} h_{i}(z(t))\left(\left(A_{i}+\Delta A_{i}(t)\right) x(t)+\left(B_{i}+\Delta B_{i}(t)\right) u(t)+F_{i} \varphi(t)\right) \\
y(t)=\sum_{i=1}^{r} h_{i}(z(t))\left(\left(C_{i}+\Delta C_{i}(t)\right) x(t)+\left(D_{i}+\Delta D_{i}(t)\right) u(t)+G_{i} \varphi(t)\right)
\end{array}\right.
$$

where $r$ represents the number of fuzzy rules. $x(t) \in \mathbb{R}^{n}, u(t) \in \mathbb{R}^{m}, y(t) \in \mathbb{R}^{q}, z(t) \in \mathbb{R}^{p}$ and $\varphi(t) \in \mathbb{R}^{d \leq n}$ represent respectively the state, the input, the output, the premisses and the external disturbances vectors. $h_{i}(z(t))$ are positive membership functions satisfying the convex sum proprieties $h_{i}(z(t)) \geq 0$ and $\sum_{i=1}^{r} h_{i}(z(t))=1 . A_{i} \in \mathbb{R}^{n \times n}, B_{i} \in \mathbb{R}^{n \times m}, C_{i} \in \mathbb{R}^{q \times n}, D_{i} \in \mathbb{R}^{q \times m}, F_{i} \in \mathbb{R}^{d \times n}, G_{i} \in \mathbb{R}^{d \times q}$ are real matrices. $\Delta A_{i}(t) \in \mathbb{R}^{n \times n}, \Delta B_{i}(t) \in \mathbb{R}^{n \times m}, \quad \Delta C_{i}(t) \in \mathbb{R}^{q \times n}$ and $\Delta D_{i}(t) \in \mathbb{R}^{q \times m}$ are Lebesgue measurable uncertainties which can be rewritten as, for $S \equiv A, B, C$ or $D, \Delta S_{i}(t)=H_{i}^{s} f_{i}^{s}(t) N_{i}^{s}$ (Zhou and Khargonekar, 1988). In that case, for the subscript $s \equiv a, b, c$ or $d$ one has $H_{i}^{s}, N_{i}^{s}$ constant matrices with appropriate dimensions and $f_{i}^{s}(t)$ uncertain matrices bounded such that $\left(f_{i}^{s}(t)\right)^{T} f_{i}^{s}(t) \leq I$.

Let us consider the following non-PDC SOFC: 
$u(t)=\left(\sum_{i=1}^{r} h_{i}(z(t)) L_{i}\right)\left(\sum_{i=1}^{r} h_{i}(z(t)) W_{i}^{5}\right)^{-1} y(t)$

where $L_{1} \in \mathbb{R}^{m \times q}$ and $W_{i}^{5} \in \mathbb{R}^{q \times q}$ are real gain matrices to be synthesized.

Remark 1: To be coherent with the aim of SOFC which is to control a dynamical system from the only knowledge of measurable signals, the premises vector $z(t)$ is required to depend only on the inputs $u(t)$, the outputs $y(t)$ and, eventually on measurable state variables. Therefore, in the case where unmeasurable variables $\bar{z}(t)$ exists in the nonlinear model to be controlled, it is still possible to report these nonlinearities from the nominal part to the uncertainties of the relevant uncertain TS model (5) to design a robust non-PDC SOFC (6) composed of membership functions $h_{i}(z(t))$ which are strictly independent of $\bar{z}(t)$. Therefore, the robust static output feedback controller design proposed in the sequel cope with unmeasurable premises variables. The benefit of uncertain TS modeling in the case of unmeasured variables will be illustrated through the example of a crane system provided in section 6.3 .

The classical way to write a closed-loop dynamics consists on substituting (6) into (5) leading, with the above defined notations, to:

$$
\begin{aligned}
\dot{x}(t)= & \left(\bar{A}_{h}+\bar{B}_{h} L_{h}\left(W_{h}^{5}\right)^{-1}\left(I-\bar{D}_{h} L_{h}\left(W_{h}^{5}\right)^{-1}\right)^{-1} \bar{C}_{h}\right) x(t) \\
& +\bar{B}_{h} L_{h}\left(W_{h}^{5}\right)^{-1}\left(I-\bar{D}_{h} L_{h}\left(W_{h}^{5}\right)^{-1}\right)^{-1} G_{h} \varphi(t)+F_{h} \varphi(t)
\end{aligned}
$$

where $\bar{A}_{h}=A_{h}+\Delta A_{h}(t), \bar{B}_{h}=B_{h}+\Delta B_{h}(t), \bar{C}_{h}=C_{h}+\Delta C_{h}(t)$ and $\bar{D}_{h}=D_{h}+\Delta D_{h}(t) y(t)$. 
Hence, the closed-loop dynamics (7) involves numerous crossing terms between the gains $L_{h}\left(W_{h}^{5}\right)^{-1}$ and the system's matrices $C_{h}, \Delta C_{h}, B_{h}, \Delta B_{h}, D_{h}$ and $\Delta D_{h}$. This leads to a strong difficulty to obtain convenient LMI conditions for the design of SOFC (6). Indeed, previous studies on SOFC design have reduced the problem by making restrictive assumptions such as $C$ common and column full rank matrices, $D_{h}=0$ and without uncertainties in the output equation (Huang and Nguang, 2006; Huang and Nguang, 2007; Chadli et al., 2002). In the sequel, we will see how this problem of crossing terms in the closed-loop dynamics may be overcame.

In (Guelton et al., 2008; Guelton et al., 2009), LMI based design for DOFC controllers has been proposed in aid of a descriptor approach. These ones are based on an interesting property called the descriptor redundancy (Tanaka et al., 2007; Bouarar et al., 2010; Chen, 2004). In the present study, taking advantage of a redundancy formulation for decoupling crossing terms occurring in (7), equations (5) and (6) can be easily rewritten as:

$$
\left\{\begin{array}{l}
\dot{x}(t)=\left(A_{h}+\Delta A_{h}(t)\right) x(t)+\left(B_{h}+\Delta B_{h}(t)\right) u(t)+F_{h} \varphi(t) \\
0 \dot{y}(t)=-y(t)+\left(C_{h}+\Delta C_{h}(t)\right) x(t)+\left(D_{h}+\Delta D_{h}(t)\right) u(t)+G_{h} \varphi(t)
\end{array}\right.
$$

and

$$
0 \dot{u}(t)=-u(t)+L_{h}\left(W_{h}^{5}\right)^{-1} y(t)
$$

Note that the redundancy consists on introducing virtual dynamics in the output equations of (8) and in 
the control law (9). Then, considering the extended state vector $\tilde{x}(t)=\left[\begin{array}{lll}x^{T}(t) & y^{T}(t) & u^{T}(t)\end{array}\right]^{T}$, the closed-loop dynamics can be expressed by the following descriptor:

$\tilde{E} \dot{\tilde{x}}(t)=\left(\tilde{A}_{h h}+\Delta \tilde{A}_{h}(t)\right) \tilde{x}(t)+\tilde{F}_{h} \varphi(t)$

with $\tilde{E}=\left[\begin{array}{lll}I & 0 & 0 \\ 0 & 0 & 0 \\ 0 & 0 & 0\end{array}\right], \tilde{A}_{h h}=\left[\begin{array}{ccc}A_{h} & 0 & B_{h} \\ C_{h} & -I & D_{h} \\ 0 & L_{h}\left(W_{h}^{5}\right)^{-1} & -I\end{array}\right], \Delta \tilde{A}_{h}(t)=\left[\begin{array}{ccc}\Delta A_{h}(t) & 0 & \Delta B_{h}(t) \\ \Delta C_{h}(t) & 0 & \Delta D_{h}(t) \\ 0 & 0 & 0\end{array}\right]$ and $\tilde{F}_{h}=\left[\begin{array}{c}F_{h} \\ G_{h} \\ 0\end{array}\right]$.

Therefore, (5) is stabilized via the control law (6) if (10) is stable. Thus, the goal is now to provide sufficient LMI stability conditions allowing to find the matrices $L_{h}$ and $W_{h}^{5}$ ensuring the stability of (10) . Unlike previous studies on static output feedbackfor continuous time TS models (Huang and Nguang, 2006; Huang and Nguang, 2007; Chadli et al., 2002) where the quadratic stability conditions are not strictly LMI, writing the closed-loop system (10) by the use of descriptor redundancy allows to avoid appearance of crossing terms between the state space matrices and the controller's ones. Therefore, the benefit of this descriptor formulation will be emphasized in the following section since it makes easier to obtain strict LMI based non-quadratic stability conditions without restrictions on the output equation of (5).

\section{LMI based conditions for SOFC design}

The main result is presented in this section. Let us first focus on the non-quadratic stabilization of uncertain TS systems (5) but without external disturbances $(\varphi(t)=0)$. 
Theorem 1: Consider, for all $k=1,2, \ldots, r, \phi_{k}$ the lower bounds of $\dot{h}_{k}(z)$. The TS fuzzy model (5) (with $\varphi(t)=0$ ) is asymptotically stable via the non-PDC SOFC (6) if there exist, for all combinations of $i=1,2, \ldots, r, 1 \leq i \neq j \leq r$ and $k=1,2, \ldots, r$, the matrices $W_{j}^{1}=\left(W_{j}^{1}\right)^{T}>0, W_{j}^{5}, W_{j}^{7}, W_{j}^{8}, W_{j}^{9}, L_{i}, R_{i j}$ and the scalars $\varepsilon_{i j}^{1 a}, \varepsilon_{i j}^{1 c}, \varepsilon_{i j}^{7 b}, \varepsilon_{i j}^{7 d}, \varepsilon_{i j}^{8 b}, \varepsilon_{i j}^{8 d}, \varepsilon_{i j}^{9 b}$ and $\varepsilon_{i j}^{9 d}$ such that the following LMI conditions are satisfied:

$\Gamma_{i i}<0$

$\frac{1}{r-1} \Gamma_{i i}+\frac{1}{2}\left(\Gamma_{i j}+\Gamma_{j i}\right)<0$

$W_{k}^{1}+R_{i j} \geq 0$

where $\Gamma_{i j}=\left[\begin{array}{cc}\tilde{\Upsilon}_{i j}+\tilde{H}_{i j} & \tilde{\mathrm{Z}}_{i j}^{T} \\ \tilde{\mathrm{Z}}_{i j} & -\tilde{\mathrm{P}}_{i j}\end{array}\right]$,

$\tilde{\Upsilon}_{i j}=\left[\begin{array}{cc}A_{i} W_{j}^{1}+W_{j}^{1} A_{i}^{T}+B_{i} W_{j}^{7}+\left(W_{j}^{7}\right)^{T} B_{i}^{T}-\sum_{k=1}^{r} \phi_{k}\left(W_{1}^{k}+R_{i j}\right) & (*) \\ \left(W_{j}^{8}\right)^{T} B_{i}^{T}+C_{i} W_{j}^{1}+D_{i} W_{j}^{7} & -W_{j}^{5}-\left(W_{j}^{5}\right)^{T}+D_{i} W_{j}^{8}+\left(W_{j}^{8}\right)^{T} D_{i}^{T} \\ \left(W_{j}^{9}\right)^{T} B_{i}^{T}-W_{j}^{7} & \left(W_{j}^{9}\right)^{T} D_{i}^{T}+L_{i}-W_{j}^{8}\end{array}\right.$

$\tilde{H}_{i j}=\left[\begin{array}{ccc}\varepsilon_{i j}^{1 a} H_{i}^{a}\left(H_{i}^{a}\right)^{T}+\left(\varepsilon_{i j}^{7 b}+\varepsilon_{i j}^{8 b}+\varepsilon_{i j}^{9 b}\right) H_{i}^{b}\left(H_{i}^{b}\right)^{T}+\varepsilon_{i j}^{1 c} H_{i}^{c}\left(H_{i}^{c}\right)^{T}+\varepsilon_{i j}^{7 d} H_{i}^{d}\left(H_{i}^{d}\right)^{T} & 0 & 0 \\ 0 & \left(\varepsilon_{i j}^{8 d}+\varepsilon_{i j}^{9 d}\right) H_{i}^{d}\left(H_{i}^{d}\right)^{T} & 0 \\ 0 & 0 & 0\end{array}\right]$ 


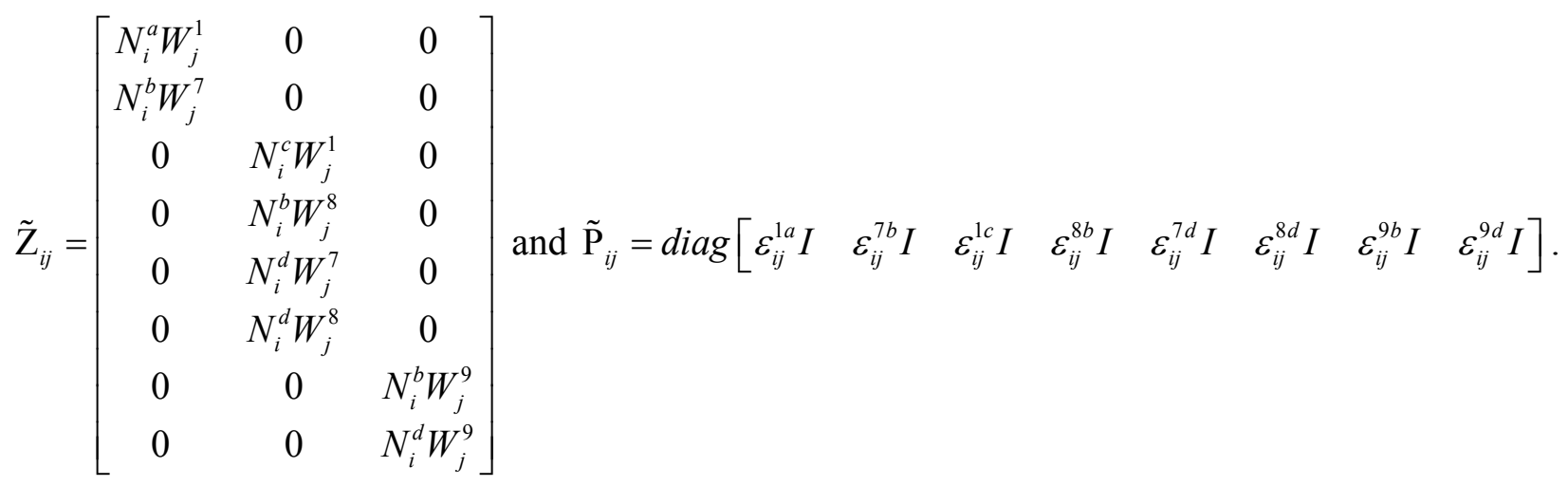

Proof: Let us consider the non-quadratic candidate fuzzy Lyapunov function given by:

$v(x(t))=\tilde{x}^{T}(t) \tilde{E}\left(\tilde{W}_{h}\right)^{-1} \tilde{x}(t)$

For (16) being a Lyapunov function one needs $v(x(t))>0, \dot{v}(x(t))<0$. Thus, classically for descriptor systems, see e.g. (Taniguchi et al., 2000; Bouarar et al., 2007), one needs:

$\tilde{E}\left(\tilde{W}_{h}\right)^{-1}=\left(\tilde{W}_{h}\right)^{-T} \tilde{E}$

Therefore, let us consider $\tilde{W}_{h}=\left[\begin{array}{ccc}W_{h}^{1} & W_{h}^{2} & W_{h}^{3} \\ W_{h}^{4} & W_{h}^{5} & W_{h}^{6} \\ W_{h}^{7} & W_{h}^{8} & W_{h}^{9}\end{array}\right]$. Multiplying (15), left by $\tilde{W}_{h}^{T}$ and right by $\tilde{W}_{h}$, one has $\tilde{W}_{h}^{T} \tilde{E}=\tilde{E} \tilde{W}_{h}$ which leads to $W_{h}^{1}=\left(W_{h}^{1}\right)^{T}>0$ (ensuring $v(x(t))>0$ ), $W_{h}^{2}=0$ and $W_{h}^{3}=0$. Then, the closed-loop system (10) is stable if:

$\dot{v}(x(t))=\dot{\tilde{x}}^{T}(t) \tilde{E}\left(\tilde{W}_{h}\right)^{-1} \tilde{x}(t)+\tilde{x}^{T}(t) \tilde{E}\left(\tilde{W}_{h}\right)^{-1} \dot{\tilde{x}}(t)+\tilde{x}^{T}(t) \tilde{E} \stackrel{\cdot}{\left.\cdot \tilde{W}_{h}\right)^{-1}} \tilde{x}(t)<0$ 
Considering (10), (16) is obviously satisfied if:

$$
\left(\tilde{A}_{h h}^{T}+\Delta \tilde{A}_{h}^{T}(t)\right)\left(\tilde{W}_{h}\right)^{-1}+\left(\tilde{W}_{h}\right)^{-T}\left(\tilde{A}_{h h}+\Delta \tilde{A}_{h}(t)\right)+\tilde{E} \widetilde{\cdot} \stackrel{\cdot\left(\tilde{W}_{h}\right)^{-1}}{<0}
$$

Multiplying left by $\tilde{W}_{h}^{T}$ and right by $\tilde{W}_{h}$ and since $\tilde{W}_{h}^{T} \tilde{E}=\tilde{E} \tilde{W}_{h}>0,(17)$ yields:

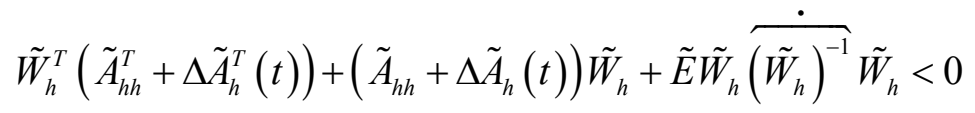

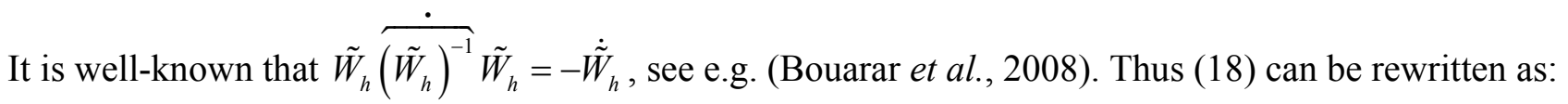

$$
\Psi_{h h h}+\Delta \Psi_{h h}(t)-\tilde{E} \dot{\tilde{W}}_{h}<0
$$

with $\Psi_{h h h}=\tilde{W}_{h}^{T} \tilde{A}_{h h}^{T}+\tilde{A}_{h h} \tilde{W}_{h}$ and $\Delta \Psi_{h h}(t)=\tilde{W}_{h}^{T} \Delta \tilde{A}_{h}^{T}(t)+\Delta \tilde{A}_{h}(t) \tilde{W}_{h}$.

Extending $\Psi_{h h h}$ with matrices defined in (10), it yields:

$$
\Psi_{h h h}=\left[\begin{array}{ccc}
A_{h} W_{h}^{1}+W_{h}^{1} A_{h}^{T}+B_{h} W_{h}^{7}+\left(W_{h}^{7}\right)^{T} B_{h}^{T} & (*) & (*) \\
\left(W_{h}^{8}\right)^{T} B_{h}^{T}+C_{h} W_{h}^{1}-W_{h}^{4}+D_{h} W_{h}^{7} & -W_{h}^{5}-\left(W_{h}^{5}\right)^{T}+D_{h} W_{h}^{8}+\left(W_{h}^{8}\right)^{T} D_{h}^{T} & (*) \\
\left(W_{h}^{9}\right)^{T} B_{h}^{T}+L_{h}\left(W_{h}^{5}\right)^{-1} W_{h}^{4}-W_{h}^{7} & -\left(W_{h}^{6}\right)^{T}+\left(W_{h}^{9}\right)^{T} D_{h}^{T}+L_{h}-W_{h}^{8} & \Psi_{h h h}^{(3,3)}
\end{array}\right]
$$


where $\Psi_{h h h}^{(3,3)}=L_{h}\left(W_{h}^{5}\right)^{-1} W_{h}^{6}+\left(W_{h}^{6}\right)^{T}\left(\left(W_{h}^{5}\right)^{-1}\right)^{T} L_{h}^{T}-W_{h}^{9}-\left(W_{h}^{9}\right)^{T}$

Let us recall that, due to the nature of the candidate Lyapunov function (14), $W_{h}^{4}, W_{h}^{5}, \ldots, W_{h}^{9}$ are slack decision matrices that are free of choice. A way to run to LMI conditions is to choose $W_{4}^{h}=0$ and $W_{6}^{h}=0$. Thus, in order to guarantee the invertibility of $\tilde{W}_{h}=\left[\begin{array}{ccc}W_{h}^{1} & 0 & 0 \\ 0 & W_{h}^{5} & 0 \\ W_{h}^{7} & W_{h}^{8} & W_{h}^{9}\end{array}\right]$ the matrices $W_{h}^{5}$ and $W_{h}^{9}$ must be non-singular and $W_{h}^{1}>0$. Consequently, (20) becomes:

$$
\bar{\Psi}_{h h}=\left[\begin{array}{ccc}
A_{h} W_{h}^{1}+W_{h}^{1} A_{h}^{T}+B_{h} W_{h}^{7}+\left(W_{h}^{7}\right)^{T} B_{h}^{T} & (*) & (*) \\
\left(W_{h}^{8}\right)^{T} B_{h}^{T}+C_{h} W_{h}^{1}+D_{h} W_{h}^{7} & -W_{h}^{5}-\left(W_{h}^{5}\right)^{T}+D_{h} W_{h}^{8}+\left(W_{h}^{8}\right)^{T} D_{h}^{T} & (*) \\
\left(W_{h}^{9}\right)^{T} B_{h}^{T}-W_{h}^{7} & \left(W_{h}^{9}\right)^{T} D_{h}^{T}+L_{h}-W_{h}^{8} & -W_{h}^{9}-\left(W_{h}^{9}\right)^{T}
\end{array}\right]
$$

Now, extending $\Delta \Psi_{h h}(t)$, it yields:

$$
\Delta \Psi_{h h}(t)=\left[\begin{array}{ccc}
\Delta \Psi_{h h}^{(1,1)}(t) & (*) & (*) \\
\Delta \Psi_{h h}^{(2,1)}(t) & \left(W_{h}^{8}\right)^{T}\left(N_{h}^{d}\right)^{T}\left(f_{h}^{d}(t)\right)^{T}\left(H_{h}^{d}\right)^{T}+H_{h}^{d} f_{h}^{d}(t) N_{h}^{d} W_{h}^{8} & (*) \\
\left(W_{h}^{9}\right)^{T}\left(N_{h}^{b}\right)^{T}\left(f_{h}^{b}(t)\right)^{T}\left(H_{h}^{b}\right)^{T} & \left(W_{h}^{9}\right)^{T}\left(N_{h}^{d}\right)^{T}\left(f_{h}^{d}(t)\right)^{T}\left(H_{h}^{d}\right)^{T} & 0
\end{array}\right]
$$

with

$$
\begin{aligned}
& \Delta \Psi_{h h}^{(1,1)}(t)=W_{h}^{1}\left(N_{h}^{a}\right)^{T}\left(f_{h}^{a}(t)\right)^{T}\left(H_{h}^{a}\right)^{T}+H_{h}^{a} f_{h}^{a}(t) N_{h}^{a} W_{h}^{1}+\left(W_{h}^{7}\right)^{T}\left(N_{h}^{b}\right)^{T}\left(f_{h}^{b}(t)\right)^{T}\left(H_{h}^{b}\right)^{T}+H_{h}^{b} f_{h}^{b}(t) N_{h}^{b} W_{h}^{7} \\
& \text { and } \Delta \Psi_{h h}^{(2,1)}(t)=\left(W_{h}^{8}\right)^{T}\left(N_{h}^{b}\right)^{T}\left(f_{h}^{b}(t)\right)^{T}\left(H_{h}^{b}\right)^{T}+H_{h}^{c} f_{h}^{c}(t) N_{h}^{c} W_{h}^{1}+H_{h}^{d} f_{h}^{d}(t) N_{h}^{d} W_{h}^{7}
\end{aligned}
$$


Expression (22) can be bounded using lemma 1 such that:

$$
\Delta \Psi_{h h}(t) \leq \Delta \bar{\Psi}_{h h}=\left[\begin{array}{ccc}
\Delta \bar{\Psi}_{h h}^{(1,1)} & (*) & (*) \\
0 & \Delta \bar{\Psi}_{h h}^{(2,2)} & (*) \\
0 & 0 & \left(\varepsilon_{h h}^{9 b}\right)^{-1}\left(W_{h}^{9}\right)^{T}\left(N_{h}^{b}\right)^{T} N_{h}^{b} W_{h}^{9}+\left(\varepsilon_{h h}^{9 d}\right)^{-1}\left(W_{h}^{9}\right)^{T}\left(N_{h}^{d}\right)^{T} N_{h}^{d} W_{h}^{9}
\end{array}\right]
$$

with:

$$
\begin{aligned}
\Delta \bar{\Psi}_{h h}^{(1,1)}= & \varepsilon_{h h}^{1 a} H_{h}^{a}\left(H_{h}^{a}\right)^{T}+\left(\varepsilon_{h h}^{7 b}+\varepsilon_{h h}^{8 b}+\varepsilon_{h h}^{9 b}\right) H_{h}^{b}\left(H_{h}^{b}\right)^{T}+\varepsilon_{h h}^{1 c} H_{h}^{c}\left(H_{h}^{c}\right)^{T}+\varepsilon_{h h}^{7 d} H_{h}^{d}\left(H_{h}^{d}\right)^{T} \\
& +\left(\varepsilon_{h h}^{1 a}\right)^{-1} W_{h}^{1}\left(N_{h}^{a}\right)^{T} N_{h}^{a} W_{h}^{1}+\left(\varepsilon_{h h}^{7 b}\right)^{-1}\left(W_{h}^{7}\right)^{T}\left(N_{h}^{b}\right)^{T} N_{h}^{b} W_{h}^{7}
\end{aligned}
$$

and

$$
\begin{aligned}
\Delta \bar{\Psi}_{h h}^{(2,2)}= & \left(\varepsilon_{h h}^{8 d}+\varepsilon_{h h}^{9 d}\right) H_{h}^{d}\left(H_{h}^{d}\right)^{T}+\left(\varepsilon_{h h}^{1 c}\right)^{-1}\left(W_{h}^{1}\right)^{T}\left(N_{h}^{c}\right)^{T} N_{h}^{c} W_{h}^{1}+\left(\varepsilon_{h h}^{8 b}\right)^{-1}\left(W_{h}^{8}\right)^{T}\left(N_{h}^{b}\right)^{T} N_{h}^{b} W_{h}^{8} \\
& +\left(\varepsilon_{h h}^{7 d}\right)^{-1}\left(W_{h}^{7}\right)^{T}\left(N_{h}^{d}\right)^{T} N_{h}^{d} W_{h}^{7}+\left(\varepsilon_{h h}^{8 d}\right)^{-1}\left(W_{h}^{8}\right)^{T}\left(N_{h}^{d}\right)^{T} N_{h}^{d} W_{h}^{8}
\end{aligned}
$$

Note that (23) can be rewritten as:

$$
\Delta \bar{\Psi}_{h h}=\tilde{H}_{h h}+\tilde{Z}_{h h}^{T}\left(\tilde{\mathrm{P}}_{h h}\right)^{-1} \tilde{\mathrm{Z}}_{h h}
$$

with

$$
\tilde{H}_{h h}=\left[\begin{array}{ccc}
\varepsilon_{h h}^{1 a} H_{h}^{a}\left(H_{h}^{a}\right)^{T}+\left(\varepsilon_{h h}^{7 b}+\varepsilon_{h h}^{8 b}+\varepsilon_{h h}^{9 b}\right) H_{h}^{b}\left(H_{h}^{b}\right)^{T}+\varepsilon_{h h}^{1 c} H_{h}^{c}\left(H_{h}^{c}\right)^{T}+\varepsilon_{h h}^{7 d} H_{h}^{d}\left(H_{h}^{d}\right)^{T} & 0 & 0 \\
0 & \left(\varepsilon_{h h}^{8 d}+\varepsilon_{h h}^{9 d}\right) H_{h}^{d}\left(H_{h}^{d}\right)^{T} & 0 \\
0 & 0 & 0
\end{array}\right]
$$




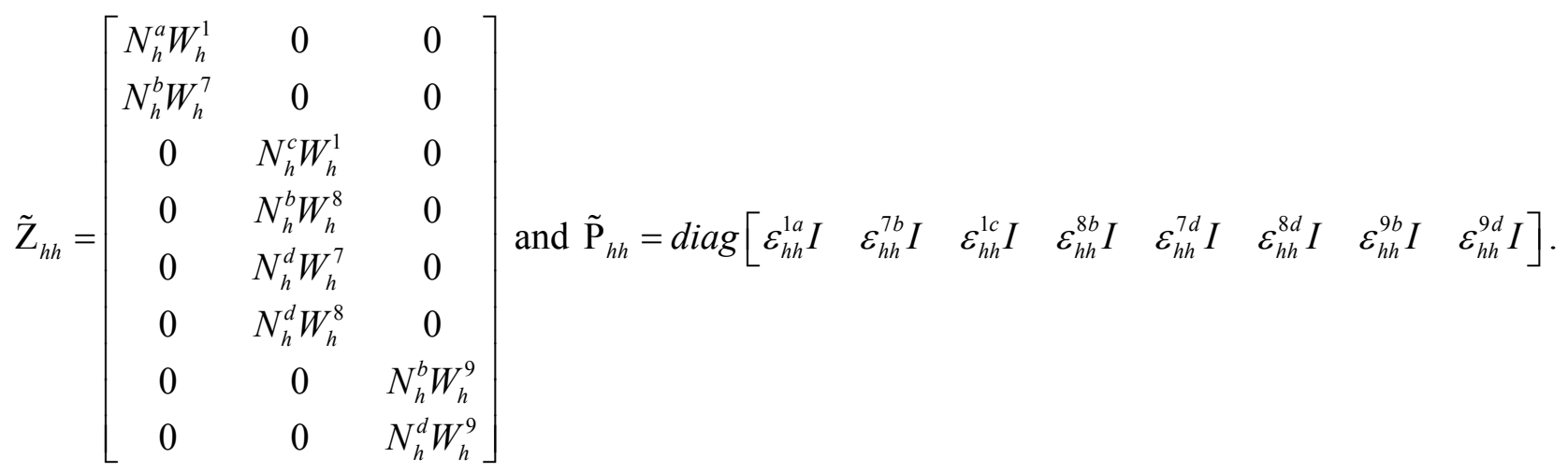

Therefore, from (21) and (24), (19) is verified if the following condition holds:

$\bar{\Psi}_{h h}+\tilde{H}_{h h}+\tilde{\mathrm{Z}}_{h h}^{T}\left(\tilde{\mathrm{P}}_{h h}\right)^{-1} \tilde{\mathrm{Z}}_{h h}-\tilde{E} \dot{\tilde{W}}_{h}<0$

Applying the Schur complement on (25), one obtains:

$\left[\begin{array}{cc}\bar{\Psi}_{h h}+\tilde{H}_{h h}-\tilde{E} \dot{\tilde{W}}_{h} & \tilde{\mathrm{Z}}_{h h}^{T} \\ \tilde{\mathrm{Z}}_{h h} & -\tilde{\mathrm{P}}_{h h}\end{array}\right]<0$

Let us now focus on $\dot{W}_{h}^{1}=\sum_{k=1}^{r} \dot{h}_{k}(z) W_{k}^{1}$ included in the term $-\tilde{E} \dot{\tilde{W}}_{h}$. From the convex property of the membership functions $h_{k}(z)$ one has $\sum_{k=1}^{r} h_{k}(z)=1$, thus $\sum_{k=1}^{r} \dot{h}_{k}(z)=0$ and so, for any fuzzy matrices $\sum_{i=1}^{r} \sum_{j=1}^{r} h_{i}(z) h_{j}(z) R_{i j}$, one has $\sum_{i=1}^{r} \sum_{j=1}^{r} \sum_{k=1}^{r} h_{i}(z) h_{j}(z) \dot{h}_{k}(z) R_{i j}=0$. Therefore, one can write:

$\dot{W}_{h}^{1}=\sum_{k=1}^{r} \dot{h}_{k}(z)\left(W_{k}^{1}+R_{i j}\right) \geq \sum_{k=1}^{r} \phi_{k}\left(W_{k}^{1}+R_{i j}\right)$ 
with, for $k=1, \ldots, r, W_{k}^{1}+R_{i j} \geq 0$ and where $\phi_{k}$ are the lower bounds of $\dot{h}_{k}(z)$.

Finally, from (26) and (27), after applying lemma 2, (26) is satisfied if the conditions proposed in theorem 1 hold. That ends the proof.

Remark 2: Equation (27) improve the proposed relaxation for non-quadratic TS based stability conditions proposed in (Mozelli et al., 2009b). Indeed, in the latter study a common slack decision variable $R$ has been introduced instead of fuzzy distributed ones $R_{i j}$, for $i=1, \ldots, r$ and $j=1, \ldots, r$. Moreover, it also improve the SOFC design conditions proposed in (Bouarar et al., 2009) for TS systems without uncertainties where a particular case of the present relaxation is considered with $R_{i j}=-W_{1}^{r}$. Note also that it can be argue that introducing fuzzy distributed slack variables may increase the number of decision variables and so the computational cost. Nevertheless, with the growing devices computational capabilities, this concern may be considered as less of a drawback and, if a solution cannot be found from theorem 1 due to computational crashes, it is still possible to check if a solution exists by setting $R_{i j}=R$ common.

\section{Hळ controller design}

This section aims at extending the previous results to the case of TS fuzzy systems with external disturbances. Hence, considering $\varphi(t) \neq 0$ and using a $H_{\infty}$ criterion, the objective is now to stabilize (5) such that the influence of the external disturbance $\varphi(t)$ on the output behavior is minimized. Let us consider the following $H_{\infty}$ criterion (Takagi and Wang, 2001): 


$$
\int_{0}^{\infty}\left(y^{T}(t) y(t)-\lambda^{2} \varphi^{T}(t) \varphi(t)\right) d t \leq 0
$$

Recall that $\tilde{x}(t)=\left[\begin{array}{lll}x^{T}(t) & y^{T}(t) & u^{T}(t)\end{array}\right]^{T}$, thus (28) can be rewritten as:

$$
\int_{0}^{\infty}\left(\tilde{x}^{T}(t) \tilde{Q} \tilde{x}(t)-\lambda^{2} \varphi^{T}(t) \varphi(t)\right) d t \leq 0
$$

with $\tilde{Q}=\left[\begin{array}{lll}0 & 0 & 0 \\ 0 & I & 0 \\ 0 & 0 & 0\end{array}\right]$.

In that case, the stability of the closed-loop system (10) is guaranteed under the constraint (29) if the LMI conditions summarized in the following theorem hold.

Theorem 2: Consider, for all $k=1,2, \ldots, r, \phi_{k}$ the lower bounds of $\dot{h}_{k}(z)$. The TS fuzzy model (5) is asymptotically stabilized via the non-PDC SOFC (6) and guarantees the attenuation level $\lambda=\sqrt{\eta}$ if there exist, for all combinations of $i=1,2, \ldots, r, 1 \leq i \neq j \leq r$ and $k=1,2, \ldots, r$, the matrices $W_{j}^{1}=\left(W_{j}^{1}\right)^{T}>0, W_{j}^{5}, W_{j}^{7}, W_{j}^{8}, W_{j}^{9}, L_{i}, R_{i j}$ and the positive scalars $\varepsilon_{i j}^{1 a}, \varepsilon_{i j}^{1 c}, \varepsilon_{i j}^{7 b}, \varepsilon_{i j}^{7 d}, \varepsilon_{i j}^{8 b}, \varepsilon_{i j}^{8 d}, \varepsilon_{i j}^{9 b}$ and $\varepsilon_{i j}^{9 d}$ such that the following LMI conditions are satisfied.

Minimize $\eta>0$ such that:

$\Theta_{i i}<0$ 
$\frac{1}{r-1} \Theta_{i i}+\frac{1}{2}\left(\Theta_{i j}+\Theta_{j i}\right)<0$

$W_{k}^{1}+R_{i j} \geq 0$

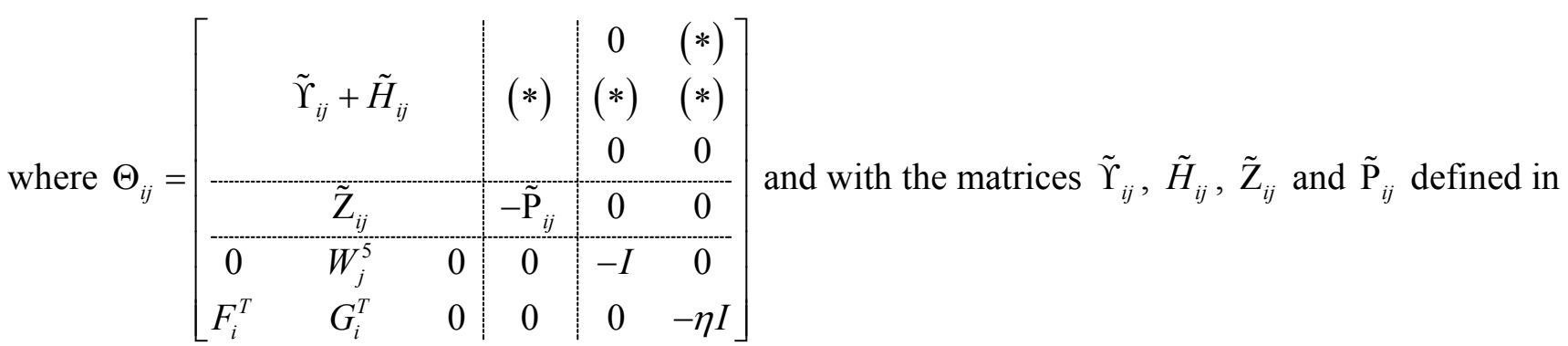
theorem 1.

Proof: The stability of the closed-loop system (10) is guaranteed, under the constraint (29), if:

$\dot{v}(x(t))+\tilde{x}^{T}(t) \tilde{Q} \tilde{x}(t)-\lambda^{2} \varphi^{T}(t) \varphi(t)<0$

That is to say if:

$$
\begin{aligned}
\tilde{x}^{T}(t)\left(\left(\tilde{A}_{h h}^{T}\right.\right. & \left.\left.+\Delta \tilde{A}_{h}^{T}(t)\right) \tilde{W}_{h}^{-1}+\left(\tilde{W}_{h}^{-1}\right)^{T}\left(\tilde{A}_{h h}+\Delta \tilde{A}_{h}(t)\right)+\tilde{E} \dot{\tilde{W}}_{h}^{-1}+\tilde{Q}\right) \tilde{x}(t) \\
& +\varphi^{T}(t) \tilde{F}_{h}^{T} \tilde{W}_{h}^{-1} \tilde{x}(t)+\tilde{x}^{T}(t)\left(\tilde{W}_{h}^{-1}\right)^{T} \tilde{F}_{h} \varphi(t)-\lambda^{2} \varphi^{T}(t) \varphi(t)<0
\end{aligned}
$$

which is obviously satisfied if: 


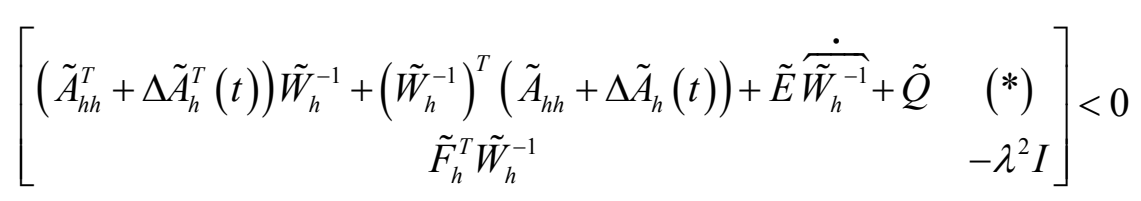

Multiplying left by $\left[\begin{array}{cc}\tilde{W}_{h}^{T} & 0 \\ 0 & I\end{array}\right]$ and right by $\left[\begin{array}{cc}\tilde{W}_{h} & 0 \\ 0 & I\end{array}\right]$, one obtains:

$$
\left[\begin{array}{cc}
W_{h}^{T}\left(\tilde{A}_{h h}^{T}+\Delta \tilde{A}_{h}^{T}(t)\right)+\left(\tilde{A}_{h h}+\Delta \tilde{A}_{h}(t)\right) W_{h}+\tilde{E} W_{h} \stackrel{\cdot}{\stackrel{\cdot \tilde{W}_{h}^{-1}}{{ }^{1}} W_{h}+W_{h}^{T} \tilde{Q} W_{h}} & (*) \\
\tilde{F}_{h}^{T} & -\lambda^{2} I
\end{array}\right]<0
$$

Following the same way as for the proof of theorem 1, (36) is satisfied if (32) holds as well as:

$$
\left[\begin{array}{cc}
\Upsilon_{h h}+\tilde{H}_{h h}+\tilde{Z}_{h h}^{T}\left(\tilde{\mathrm{P}}_{h h}\right)^{-1} \tilde{\mathrm{Z}}_{h h}+W_{h}^{T} \tilde{Q} W_{h} & (*) \\
\tilde{F}_{h}^{T} & -\lambda^{2} I
\end{array}\right]<0
$$

Note that $\tilde{W}_{h}^{T} \tilde{Q} \tilde{W}_{h}=\left[\begin{array}{ccc}0 & 0 & 0 \\ 0 & \left(W_{h}^{5}\right)^{T} W_{h}^{5} & 0 \\ 0 & 0 & 0\end{array}\right]$, using the Schur complement and lemma 2, (30) and (31) yield. That ends the proof.

\section{Discussion on non-quadratic approaches limits:}

The LMI conditions proposed in theorems 1 and 2 depend on the lower bounds of $\dot{h}_{k}(z)$ for $k=1, \ldots, r$. Even if it is often pointed out as a criticism to fuzzy Lyapunov approaches since these parameters may be difficult to choose, a way to obtain these bounds has been proposed in (Tanaka et al., 2003) in some special cases. Moreover, let us recall that this approach remains one of the least conservative in terms of 
LMI based design (Mozelli et al., 2009b). In (Tanaka et al., 2007; Guerra et al., 2007; Guelton et al., 2009), a fuzzy Lyapunov candidate function has been reduced leading to relaxed quadratic stability. Indeed, some elements in the Lyapunov matrix can be set common in order to make the LMIs free of membership function's lower bounds. In the present study, this remains on setting $W_{1}$ common matrices in the previous theorems. However, following the latter way, the 'price' to pay for more practical applicability is obviously an increase of the conservatism. An elegant way has also been recently proposed to overcome the knowledge of the membership function derivative bounds in (Bernal and Guerra, 2010; Guerra et al., 2012). However, in these studies, the design goal has been reduced to a local view point and lead to complex LMI formulation which are, at this time only available for standard stability analysis and stabilization. Another approach based on line integral Lyapunov functions, which is not investigated in this paper, has been proposed to avoid appearance of membership function derivatives in non-quadratic stability conditions (Rhee and Won, 2006; Guelton et al., 2010, Mozelli 2009a). Therefore, some further research efforts will have to be done to extend these approaches to SOFC design. However, Let us point out that the goal of the present study is not to reach the difficulties of non-quadratic approaches but to overcome and derive LMI based SOFC design for a general class of TS systems (5) without constraining assumptions on output equation and including the most commonly used and effective non-quadratic approach for conservatism reduction.

\section{Numerical examples}

In this section, in order to show the efficiency and the applicability of the proposed fuzzy approaches, three examples are considered. The aim of the first one is to compare the conservatism of the approach proposed in theorem 2 without uncertainties, the quadratic stability conditions discussed in the above section ( $W_{1}$ set as a common matrix in theorem 1 and 2 ) and the non-quadratic conditions proposed in our preliminary study (Bouarar et al., 2009) through a numerical example. Then, a second example is 
devoted to show the effectiveness of the SOFC based design on a $4^{\text {th }}$ order and 4 rules numerical uncertain and disturbed TS system containing nonlinearities in the output equation. Finally, the benchmark of a crane system is considered in simulation as a third example to illustrate the validity of the proposed approach on an engineering application with unmeasurable premises variables reported as uncertainties.

\subsection{Example 1:}

Let us consider the following uncertain and disturbed TS fuzzy model:

$$
\left\{\begin{array}{l}
\dot{x}(t)=\sum_{i=1}^{2} h_{i}(z(t))\left(A_{i} x(t)+B_{i} u(t)+F_{i} \varphi(t)\right) \\
y(t)=\sum_{i=1}^{2} h_{i}(z(t))\left(C_{i} x(t)+D_{i} u(t)+G_{i} \varphi(t)\right)
\end{array}\right.
$$

with $\quad A_{1}=\left[\begin{array}{ll}-5 & -4 \\ -1 & -2\end{array}\right], \quad A_{2}=\left[\begin{array}{cc}-2 & -4 \\ 10 & -2\end{array}\right], \quad B_{1}=\left[\begin{array}{c}0 \\ 10\end{array}\right], \quad B_{2}=\left[\begin{array}{l}0 \\ 3\end{array}\right], \quad C_{1}=\left[\begin{array}{cc}2 & -10 \\ 5 & -1\end{array}\right], \quad C_{2}=\left[\begin{array}{cc}-3 & 20 \\ -7 & -2\end{array}\right]$, $D_{1}=\left[\begin{array}{c}3 \\ -1\end{array}\right], \quad D_{2}=\left[\begin{array}{c}-2 \\ 0.5\end{array}\right], \quad F_{1}=F_{2}=\left[\begin{array}{c}0 \\ 0.25\end{array}\right], \quad G_{1}=\left[\begin{array}{c}-0.5 \\ 0.5\end{array}\right], \quad x(t)=\left[\begin{array}{ll}x_{1}(t) & \left.x_{2}(t)\right]^{T},\end{array}\right.$ $y(t)=\left[\begin{array}{ll}y_{1}(t) & y_{2}(t)\end{array}\right]^{T}, z(t)=y_{1}(t)$ and $h_{1}(z(t))=\cos ^{2}\left(y_{1}(t)\right)=1-h_{2}(z(t))$.

Using the Matlab LMI Toolbox, the attenuation level value corresponding to the quadratic approach ( $W_{1}$ set as common matrix and $R_{i j}=-W_{1}$ in theorem 2) is $\lambda=1.2758$. This solution can be improved since the non-quadratic LMI conditions are reputed of less conservatism. Nevertheless, in that case, the respectively lower bounds $\phi_{1}$ and $\phi_{2}$ of $\dot{h}_{1}(z)$ and $\dot{h}_{2}(z)$, which are difficult to choose in practice, are required. For the sake of generality, one proposes to study the influence of these bounds on the 
conservatism of the proposed LMI conditions regarding to the above discussed quadratic result and the non-quadratic conditions proposed in our preliminary study (Bouarar et al., 2009). Thus, the attenuation level has been computed from theorem 2 of the present study and theorem 2 in (Bouarar et al., 2009) for $\phi_{2}=-1$ and $\phi_{1} \in\left[\begin{array}{ll}-4 & -0.5\end{array}\right]$. These results are summarized in Fig. 1. From the latter, one can conclude that, to stabilize (38) without uncertainties, the proposed non-quadratic approach (theorem 2) leads to the lower conservatism results since the $H_{\infty}$ performances are improved.

\subsection{Example 2:}

Let us consider the following $4^{\text {th }}$ order-4 rules uncertain and disturbed TS system with nonlinear output equation:

$$
\left\{\begin{array}{l}
\dot{x}(t)=\sum_{i=1}^{4} h_{i}(z(t))\left(\left(A_{i}+\Delta A_{i}(t)\right) x(t)+\left(B_{i}+\Delta B_{i}(t)\right) u(t)+F_{i} \varphi(t)\right) \\
y(t)=\sum_{i=1}^{4} h_{i}(z(t))\left(\left(C_{i}+\Delta C_{i}(t)\right) x(t)+\left(D_{i}+\Delta D_{i}(t)\right) u(t)+G_{i} \varphi(t)\right)
\end{array}\right.
$$

where $\quad x(t)=\left[\begin{array}{llll}x_{1}(t) & x_{2}(t) & x_{3}(t) & x_{4}(t)\end{array}\right]^{T}, \quad y(t)=\left[\begin{array}{llll}y_{1}(t) & y_{2}(t) & y_{3}(t) & y_{4}(t)\end{array}\right]^{T}, \quad z(t) \equiv y_{1}(t)$,

$$
h_{1}(z(t))=\frac{1+\sin \left(y_{1}(t)\right)}{2}, \quad h_{2}(z(t))=\frac{1+\cos \left(y_{1}(t)\right)}{2}, \quad h_{3}(z(t))=\frac{\cos ^{2}\left(y_{1}(t)\right)-1}{2}
$$

$h_{4}(z(t))=1-\left(h_{1}(z(t))+h_{2}(z(t))+h_{3}(z(t))\right)$ and the nominal system's matrices:

$$
A_{1}=\left[\begin{array}{cccc}
-10 & 0.5 & -1 & 0 \\
0.5 & -3 & 0 & -1 \\
1 & 1 & -4 & -1 \\
0.5 & -1 & 0 & -6
\end{array}\right], A_{2}=\left[\begin{array}{cccc}
-3 & -1 & -1 & 0 \\
1 & -3 & -1.5 & 0.5 \\
1.5 & 0 & -3.5 & 0 \\
-0.5 & 0.5 & 0 & -8
\end{array}\right], A_{3}=\left[\begin{array}{cccc}
-5 & 0 & 1 & 0 \\
0.2 & -4 & 0 & 0 \\
1.5 & 0.5 & -1 & -1 \\
0.55 & -0.5 & 0 & -4
\end{array}\right],
$$




$$
\begin{aligned}
& A_{4}=\left[\begin{array}{cccc}
-15 & 0.5 & 1 & 0 \\
0 & -5 & -2.5 & 0 \\
0.5 & 0 & -1.5 & 0 \\
-1 & 0.4 & 0 & -7
\end{array}\right], B_{1}=\left[\begin{array}{c}
2.5 \\
1 \\
2.8 \\
0
\end{array}\right], B_{2}=\left[\begin{array}{c}
1.5 \\
0.5 \\
-5.5 \\
0
\end{array}\right], B_{3}=\left[\begin{array}{c}
0.5 \\
0 \\
1 \\
0
\end{array}\right], B_{4}=\left[\begin{array}{c}
-0.5 \\
0.15 \\
-5.5 \\
0.5
\end{array}\right], C_{1}=\left[\begin{array}{cccc}
-2 & 1 & 0 & 0 \\
0 & -3 & 0 & 0 \\
-1 & -0.5 & 0 & 0 \\
0 & -1 & 0 & 0
\end{array}\right], \\
& C_{2}=\left[\begin{array}{cccc}
3 & 2 & 0 & 0 \\
-1 & -2 & 0 & 0 \\
-5.5 & 0.5 & 0 & 0 \\
0.5 & -1 & 0 & 0
\end{array}\right], \quad C_{3}=\left[\begin{array}{cccc}
-2.5 & 1.5 & 0 & 0 \\
0 & -1.5 & 0 & 0 \\
-2 & 1 & 0 & 0 \\
0.5 & -2 & 0 & 0
\end{array}\right], \quad C_{4}=\left[\begin{array}{cccc}
1 & 0.8 & 0 & 0 \\
0 & -0.5 & 0 & 0 \\
-5.5 & 0.6 & 0 & 0 \\
2.5 & 2 & 0 & 0
\end{array}\right], \quad D_{1}=\left[\begin{array}{l}
0.3 \\
0.1 \\
0.1 \\
0.1
\end{array}\right], \\
& D_{2}=\left[\begin{array}{c}
0.1 \\
-0.2 \\
0 \\
0
\end{array}\right], \quad D_{3}=\left[\begin{array}{c}
0.15 \\
0.2 \\
0.25 \\
0
\end{array}\right], \quad D_{4}=\left[\begin{array}{c}
0.35 \\
-0.4 \\
0.15 \\
0
\end{array}\right], \quad F_{1}=\left[\begin{array}{c}
0 \\
-0.25 \\
0 \\
0
\end{array}\right], \quad F_{2}=\left[\begin{array}{c}
-0.25 \\
0.25 \\
0 \\
0
\end{array}\right], \quad F_{3}=\left[\begin{array}{c}
0.2 \\
-0.5 \\
0.2 \\
0
\end{array}\right], \quad F_{4}=\left[\begin{array}{c}
-0.5 \\
0.2 \\
0.6 \\
0
\end{array}\right], \\
& G_{1}=\left[\begin{array}{c}
-0.3 \\
0.3 \\
0 \\
0
\end{array}\right], G_{2}=\left[\begin{array}{c}
0.35 \\
0.2 \\
0.1 \\
0
\end{array}\right], G_{3}=\left[\begin{array}{c}
-0.2 \\
0.35 \\
0 \\
0.2
\end{array}\right], G_{4}=\left[\begin{array}{c}
0.3 \\
0 \\
0.45 \\
0.1
\end{array}\right] \text {, }
\end{aligned}
$$

as well as the uncertain matrices $\Delta A_{i}(t)=H_{i}^{a} f_{a}(t) N_{i}^{a}, \Delta B_{i}(t)=H_{i}^{b} f_{b}(t) N_{i}^{b}, \Delta C_{i}(t)=H_{i}^{c} f_{c}(t) N_{i}^{c}$ and $\Delta D_{i}(t)=H_{i}^{d} f_{d}(t) N_{i}^{d}$ with:

$$
\begin{aligned}
& H_{1}^{a}=\left[\begin{array}{c}
0 \\
0.1 \\
0.25 \\
0.1
\end{array}\right], \quad H_{2}^{a}=\left[\begin{array}{c}
0 \\
-0.1 \\
0.15 \\
0.1
\end{array}\right], \quad H_{3}^{a}=\left[\begin{array}{c}
0 \\
0.15 \\
0.05 \\
0.1
\end{array}\right], H_{4}^{a}=\left[\begin{array}{c}
0 \\
-0.2 \\
0.25 \\
0.3
\end{array}\right], \quad H_{1}^{b}=\left[\begin{array}{c}
0 \\
-0.2 \\
0 \\
0
\end{array}\right], \quad H_{2}^{b}=\left[\begin{array}{c}
0 \\
-0.1 \\
0 \\
0
\end{array}\right], \quad H_{3}^{b}=\left[\begin{array}{c}
0 \\
0.25 \\
0 \\
0
\end{array}\right], \\
& H_{4}^{b}=\left[\begin{array}{c}
0 \\
0.2 \\
0 \\
0.1
\end{array}\right], \quad H_{1}^{c}=\left[\begin{array}{c}
0.1 \\
-0.1 \\
0 \\
0.1
\end{array}\right], \quad H_{2}^{c}=\left[\begin{array}{c}
-0.1 \\
0.1 \\
0 \\
0.1
\end{array}\right], \quad H_{3}^{c}=\left[\begin{array}{c}
0 \\
0.2 \\
0 \\
0
\end{array}\right], \quad H_{4}^{c}=\left[\begin{array}{c}
-0.2 \\
0.15 \\
0 \\
0
\end{array}\right], \quad H_{1}^{d}=\left[\begin{array}{c}
0.5 \\
-0.5 \\
0 \\
0.2
\end{array}\right], \quad H_{2}^{d}=\left[\begin{array}{c}
-0.1 \\
0.1 \\
0.1 \\
0.1
\end{array}\right], \\
& H_{3}^{d}=\left[\begin{array}{c}
-0.5 \\
0 \\
0.25 \\
0.1
\end{array}\right], \quad H_{4}^{d}=\left[\begin{array}{c}
-0.25 \\
0.2 \\
0.3 \\
0
\end{array}\right], \quad N_{1}^{a}=\left[\begin{array}{llll}
0.1 & 0.1 & -0.1 & 0.2
\end{array}\right], \quad N_{2}^{a}=\left[\begin{array}{llll}
-0.1 & 0.1 & 0.1 & -0.2
\end{array}\right] \text {, }
\end{aligned}
$$

$N_{3}^{a}=\left[\begin{array}{llll}-0.1 & -0.1 & 0.1 & 0\end{array}\right], N_{1}^{a}=\left[\begin{array}{llll}0 & -0.2 & 0.3 & -0.4\end{array}\right], N_{1}^{b}=0.1, N_{2}^{b}=-0.175, N_{3}^{b}=0.15, N_{4}^{b}=-0.25$, 


$$
\begin{aligned}
& N_{1}^{c}=\left[\begin{array}{llll}
0.1 & 0.1 & 0.1 & 0.15
\end{array}\right], \quad N_{2}^{c}=\left[\begin{array}{llll}
-0.1 & -0.1 & 0.1 & -0.2
\end{array}\right], \quad N_{3}^{c}=\left[\begin{array}{llll}
0.2 & -0.1 & 0 & -0.15
\end{array}\right], \\
& N_{4}^{c}=\left[\begin{array}{llll}
-0.2 & -0.15 & 0.15 & -0.25
\end{array}\right], N_{1}^{d}=-0.15, N_{2}^{d}=0.05, N_{3}^{d}=-0.25, N_{4}^{d}=0.15 \text {. }
\end{aligned}
$$

The goal is to design a robust $H_{\infty} K \infty$ controller that guarantee the attenuation of the external disturbances $\varphi(t)$ affecting the system output and state vector. The following SOFC gain matrices, guaranteeing a minimized $H_{\infty}$ attenuation level $\lambda=0.8533$, are obtained using the Matlab LMI Toolbox through theorem 2 with the prescribed lower bounds values $\phi_{1}=-1, \phi_{2}=-0.8, \phi_{3}=-2, \phi_{4}=-1.5$.

$$
\begin{aligned}
& W_{1}^{5}=\left[\begin{array}{cccc}
1.3685 & 0.0901 & 0.0221 & -0.0121 \\
0.0901 & 1.1756 & -0.0078 & -0.0488 \\
0.0221 & -0.0078 & 1.4464 & -0.0746 \\
-0.0121 & -0.0488 & -0.0746 & 1.4055
\end{array}\right], W_{2}^{5}=\left[\begin{array}{cccc}
1.2426 & 0.0375 & -0.0137 & 0.0422 \\
0.0375 & 1.4033 & -0.0236 & 0.0112 \\
-0.0137 & -0.0236 & 1.1421 & 0.0506 \\
0.0422 & 0.0112 & 0.0506 & 1.3595
\end{array}\right] \\
& W_{3}^{5}=\left[\begin{array}{cccc}
1.1476 & 0.1207 & -0.0695 & -0.0071 \\
0.1207 & 1.2730 & 0.0722 & -0.1092 \\
-0.0695 & 0.0722 & 1.4095 & 0.0409 \\
-0.0071 & -0.1092 & 0.0409 & 1.1276
\end{array}\right], W_{4}^{5}=\left[\begin{array}{cccc}
1.3887 & -0.0662 & -0.0462 & -0.1025 \\
-0.0662 & 1.4318 & -0.0098 & 0.0479 \\
-0.0462 & -0.0098 & 1.2250 & -0.0237 \\
-0.1025 & 0.0479 & -0.0237 & 1.3264
\end{array}\right] \text {, } \\
& L_{1}=\left[\begin{array}{llll}
-0.0842 & 0.218 & 0.0701 & 0.0352
\end{array}\right], L_{2}=\left[\begin{array}{llll}
-0.1448 & 0.116 & 0.1203 & -0.0019
\end{array}\right], \\
& L_{3}=\left[\begin{array}{llll}
0.2542 & -0.2131 & 0.0919 & -0.2372
\end{array}\right] \text { and } L_{4}=\left[\begin{array}{lllll}
-0.0086 & 0.0368 & 0.006 & -0.0153
\end{array}\right] \text {. }
\end{aligned}
$$

For simulation realization, the disturbance signal $\varphi(t)$ have been chosen as a white noise with unit variance characterized by a bounded energy added to a step (amplitude 0.5 for $t \in[5 s, 10 s]$, 0 elsewhere). Moreover, the uncertain variables have been set as $f_{a}(t)=0.25 \sin (0.5 t)$, $f_{b}(t)=0.3 \cos (2 t), f_{c}(t)=0.2 \frac{\sin (\pi t)}{\pi t}, f_{d}(t)=0.15 \cos ^{2}(2 t)$. Fig 2, 3 and 4 show respectively the system's state and output responses, the control signal and the disturbance evolutions for the initial conditions $x(0)=\left[\begin{array}{llll}0.5 & 0.3 & 0.5 & -0.5\end{array}\right]^{T}$ and $y(0)=\left[\begin{array}{llll}-0.5 & 0.5 & 0.5 & -0.5\end{array}\right]^{T}$. The synthesized robust 
SOFC is correctly stabilizing the system and attenuates the external disturbances effect with a minimized $H_{\infty}$ attenuation level $\lambda=0.8533$.

\subsection{Example 3:}

Let us consider the crane system depicted in figure 5 with the parameters and variables given in table 1 . From the well-known Lagrange formalism, the motion equations of the crane system are given by:

$$
\left\{\begin{array}{l}
a \ddot{r}(t)+c \ddot{\theta}(t) \cos \theta(t)-c \dot{\theta}^{2}(t) \sin \theta(t)+k_{s} r(t)+k_{d} \dot{r}(t)=u(t)+\varphi_{1}(t) \\
b \ddot{\theta}(t)+c \ddot{r}(t) \cos \theta(t)+c g \sin \theta(t)=\varphi_{2}(t)
\end{array}\right.
$$

with $a=m+M, b=m L^{2}+I_{y y}, c=m L$ and where $\varphi_{1}(t)$ and $\varphi_{2}(t)$ are external disturbance, i.e. respectively an external force vector on the cart and an external torque on the pendulum and the parameters given table 1 .

By considering the inertia matrix $M(\theta)=\left[\begin{array}{cc}a & c \cos \theta \\ c \cos \theta & b\end{array}\right]$ and its inverse $M^{-1}(\theta)=\frac{1}{a b-c^{2} \cos ^{2} \theta}\left[\begin{array}{cc}b & -c \cos \theta \\ -c \cos \theta & a\end{array}\right],(40)$ can be rewritten as:

$\left\{\begin{array}{l}\ddot{r}=\frac{1}{a b-c^{2} \cos ^{2} \theta}\left(-b k_{s} r+b c \dot{\theta}^{2} \sin \theta+c^{2} g \cos \theta \sin \theta-k_{d} b \dot{r}+b u+b \varphi_{1}-c \varphi_{2} \cos \theta\right) \\ \ddot{\theta}=\frac{1}{a b-c^{2} \cos ^{2} \theta}\left(c k_{s} r \cos \theta-a c g \sin \theta-c^{2} \dot{\theta}^{2} \cos \theta \sin \theta+c k_{d} \dot{r} \cos \theta-c u \cos \theta-c \varphi_{1} \cos \theta+a \varphi_{2}\right)\end{array}\right.$

Note that the robot's velocities $\dot{r}(t)$ and $\dot{\theta}(t)$ aren't considered available for measurement. Therefore, 
from the dynamical model (41) and to cope with the goal of designing a robust non-PDC SOFC which doesn't require unmeasurable premise variables (see remark 1), the nonlinear terms $b c \dot{\theta}^{2}(t) \sin \theta(t)$ and $-c^{2} \dot{\theta}^{2}(t) \cos \theta(t) \sin \theta(t)$ will be cast as uncertainties. Moreover, the motion of the system being physically restricted, the angular velocity can be bounded such that $|\dot{\theta}(t)|<\alpha$ and so one can denote the nonlinear term $\delta(\dot{\theta}(t))=\frac{\dot{\theta}^{2}(t)}{\alpha^{2}}, \quad$ normalized such that $\quad \delta^{2}(\dot{\theta}(t)) \leq 1$. Then, let $x(t)=\left[\begin{array}{llll}r(t) & \theta(t) & \dot{r}(t) & \dot{\theta}(t)\end{array}\right]^{T}$ be the state vector of the crane, $y(t)=q=\left[\begin{array}{ll}r(t) & \theta(t)\end{array}\right]^{T}$ the output vector, $\varphi(t)=\left[\begin{array}{lll}\varphi_{1}(t) & \varphi_{2}(t)\end{array}\right]^{T}$ the vector of external disturbances. From (41) an uncertain state space model is given by:

$\left\{\begin{array}{l}\dot{x}(t)=(A(\theta(t))+\Delta A(\theta(t), \delta(\dot{\theta}(t)))) x(t)+B(\theta(t)) u(t)+F(\theta(t)) \varphi(t) \\ y(t)=C x(t)\end{array}\right.$

with $A(\theta)=\eta_{3}(\theta)\left[\begin{array}{cccc}0 & 0 & 1 & 0 \\ 0 & 0 & 0 & 1 \\ -k_{s} b & c^{2} g \eta_{1}(\theta) \eta_{2}(\theta) & -k_{d} b & 0 \\ k_{s} c \eta_{1}(\theta) & -a c g \eta_{2}(\theta) & k_{d} c \eta_{1}(\theta) & 0\end{array}\right]$,

$\Delta A(\theta, \delta(\dot{\theta}))=\eta_{3}(\theta)\left[\begin{array}{cccc}0 & 0 & 0 & 0 \\ 0 & 0 & 0 & 0 \\ 0 & b c \alpha^{2} \delta(\dot{\theta}) \eta_{2}(\theta) & 0 & 0 \\ 0 & -c^{2} \alpha^{2} \delta(\dot{\theta}) \eta_{1}(\theta) \eta_{2}(\theta) & 0 & 0\end{array}\right]=H_{a}(\theta) \Delta_{a}(t) N_{a}, \Delta_{a}(t)=\delta(\dot{\theta}), N_{a}=\left[\begin{array}{l}0 \\ 1 \\ 0 \\ 0\end{array}\right]^{T}$,

$H_{a}(\theta)=\eta_{3}(\theta)\left[\begin{array}{c}0 \\ 0 \\ b c \alpha^{2} \eta_{2}(\theta) \\ -c^{2} \alpha^{2} \eta_{1}(\theta) \eta_{2}(\theta)\end{array}\right], \quad B(\theta)=\eta_{3}(\theta)\left[\begin{array}{c}0 \\ 0 \\ b \\ -c \eta_{1}(\theta)\end{array}\right], \quad F(\theta)=\eta_{3}(\theta)\left[\begin{array}{cc}0 & 0 \\ 0 & 0 \\ b & -c \eta_{1}(\theta) \\ -c \eta_{1}(\theta) & a\end{array}\right]$, 
$C=\left[\begin{array}{llll}1 & 0 & 0 & 0 \\ 0 & 1 & 0 & 0\end{array}\right]$ and where, for $\theta \in\left[-\theta_{0}, \theta_{0}\right], \quad \eta_{1}(\theta)=\cos \theta \in[\beta, 1] \quad$ with $\beta=\cos \theta_{0}$, $\eta_{2}(\theta)=\frac{\sin \theta}{\theta} \in[\rho, 1]$ with $\rho=\frac{\sin \theta_{0}}{\theta_{0}}$, and $\eta_{3}(\theta)=\frac{1}{a b-c^{2} \cos ^{2} \theta} \in\left[\frac{1}{a b-c^{2}}, \frac{1}{a b-c^{2} \beta^{2}}\right]$ are bounded nonlinearities.

Therefore, one may apply the sector nonlinearity approach (Tanaka \& Wang, 2001) such that:

$$
\begin{aligned}
& \eta_{1}(\theta)=\underbrace{\frac{\eta_{1}(\theta)-\beta}{1-\beta}}_{w_{11}(\theta(t))}(1)+\underbrace{\frac{1-\eta_{1}(\theta)}{1-\beta}}_{w_{12}(\theta(t))}(\beta) \\
& \eta_{2}(\theta)=\underbrace{\frac{\eta_{2}(\theta)-\rho}{1-\rho}}_{w_{21}(\theta(t))}(1)+\underbrace{\frac{1-\eta_{2}(\theta)}{1-\rho}}_{w_{22}(\theta(t))}(\rho) \\
& \eta_{3}(\theta)=\underbrace{\frac{\left(a b-c^{2} \beta^{2}\right)\left(\left(a b-c^{2}\right) \eta_{3}(\theta)-1\right)}{\beta_{31}}\left(\frac{1}{a b-c^{2} \beta^{2}}\right)+\underbrace{\frac{\left(a b-c^{2}\right)\left(1-\left(a b-c^{2} \beta^{2}\right) \eta_{2}(\theta)\right)}{c^{2}\left(\beta^{2}-1\right)}}_{w_{32}\left(\theta_{1}(t)\right)}\left(\frac{1}{a b-c^{2}}\right)}_{c^{2}\left(\beta^{2}-1\right)}
\end{aligned}
$$

leading to define the 8 following membership functions as $h_{1}(\theta)=w_{11}(\theta) w_{21}(\theta) w_{31}(\theta)$, $h_{2}(\theta)=w_{11}(\theta) w_{21}(\theta) w_{32}(\theta), \quad h_{3}(\theta)=w_{11}(\theta) w_{22}(\theta) w_{31}(\theta), \quad h_{4}(\theta)=w_{11}(\theta) w_{22}(\theta) w_{32}(\theta)$, $h_{5}(\theta)=w_{12}(\theta) w_{21}(\theta) w_{31}(\theta), \quad h_{6}(\theta)=w_{12}(\theta) w_{21}(\theta) w_{32}(\theta), \quad h_{7}(\theta)=w_{12}(\theta) w_{22}(\theta) w_{31}(\theta)$, $h_{8}(\theta)=w_{12}(\theta) w_{22}(\theta) w_{32}(\theta)$ and the relevant 8 rules uncertain T-S model of the crane given by:

$\left\{\begin{array}{l}\dot{x}(t)=\sum_{i=1}^{8} h_{i}(\theta(t))\left(\left(A_{i}+\Delta A_{i}(t)\right) x(t)+B_{i} u(t)+F_{i} \varphi(t)\right) \\ y(t)=C x(t)\end{array}\right.$ 
with $A_{1}=\left[\begin{array}{cccc}0 & 0 & 1 & 0 \\ 0 & 0 & 0 & 1 \\ -\frac{k_{s} b}{a b-c^{2} \beta^{2}} & \frac{c^{2} g}{a b-c^{2} \beta^{2}} & -\frac{k_{d} b}{a b-c^{2} \beta^{2}} & 0 \\ \frac{k_{s} c}{a b-c^{2} \beta^{2}} & -\frac{a c g}{a b-c^{2} \beta^{2}} & \frac{k_{d} c}{a b-c^{2} \beta^{2}} & 0\end{array}\right], A_{2}=\left[\begin{array}{cccc}0 & 0 & 1 & 0 \\ 0 & 0 & 0 & 1 \\ -\frac{k_{s} b}{a b-c^{2}} & \frac{c^{2} g}{a b-c^{2}} & -\frac{k_{d} b}{a b-c^{22}} & 0 \\ \frac{k_{s} c}{a b-c^{2}} & -\frac{a c g}{a b-c^{2}} & \frac{k_{d} c}{a b-c^{2}} & 0\end{array}\right]$,

$A_{3}=\left[\begin{array}{cccc}0 & 0 & 1 & 0 \\ 0 & 0 & 0 & 1 \\ -\frac{k_{s} b}{a b-c^{2} \beta^{2}} & \frac{c^{2} g \rho}{a b-c^{2} \beta^{2}} & -\frac{k_{d} b}{a b-c^{2} \beta^{2}} & 0 \\ \frac{k_{s} c}{a b-c^{2} \beta^{2}} & -\frac{a c g \rho}{a b-c^{2} \beta^{2}} & \frac{k_{d} c}{a b-c^{2} \beta^{2}} & 0\end{array}\right], A_{4}=\left[\begin{array}{cccc}0 & 0 & 1 & 0 \\ 0 & 0 & 0 & 1 \\ -\frac{k_{s} b}{a b-c^{2}} & \frac{c^{2} g \rho}{a b-c^{2}} & -\frac{k_{d} b}{a b-c^{2}} & 0 \\ \frac{k_{s} c}{a b-c^{2}} & -\frac{a c g \rho}{a b-c^{2}} & \frac{k_{d} c}{a b-c^{2}} & 0\end{array}\right]$,

$A_{5}=\left[\begin{array}{cccc}0 & 0 & 1 & 0 \\ 0 & 0 & 0 & 1 \\ -\frac{k_{s} b}{a b-c^{2} \beta^{2}} & \frac{c^{2} g \beta}{a b-c^{2} \beta^{2}} & -\frac{k_{d} b}{a b-c^{2} \beta^{2}} & 0 \\ \frac{k_{s} c \beta}{a b-c^{2} \beta^{2}} & -\frac{a c g}{a b-c^{2} \beta^{2}} & \frac{k_{d} c \beta}{a b-c^{2} \beta^{2}} & 0\end{array}\right], A_{6}=\left[\begin{array}{cccc}0 & 0 & 1 & 0 \\ 0 & 0 & 0 & 1 \\ -\frac{k_{s} b}{a b-c^{2}} & \frac{c^{2} g \beta}{a b-c^{2}} & -\frac{k_{d} b}{a b-c^{2}} & 0 \\ \frac{k_{s} c \beta}{a b-c^{2}} & -\frac{a c g}{a b-c^{2}} & \frac{k_{d} c \beta}{a b-c^{2}} & 0\end{array}\right]$,

$A_{7}=\left[\begin{array}{cccc}0 & 0 & 1 & 0 \\ 0 & 0 & 0 & 1 \\ -\frac{k_{s} b}{a b-c^{2} \beta^{2}} & \frac{c^{2} g \beta \rho}{a b-c^{2} \beta^{2}} & -\frac{k_{d} b}{a b-c^{2} \beta^{2}} & 0 \\ \frac{k_{s} c \beta}{a b-c^{2} \beta^{2}} & -\frac{a c g \rho}{a b-c^{2} \beta^{2}} & \frac{k_{d} c \beta}{a b-c^{2} \beta^{2}} & 0\end{array}\right], A_{8}=\left[\begin{array}{cccc}0 & 0 & 1 & 0 \\ 0 & 0 & 0 & 1 \\ -\frac{k_{s} b}{a b-c^{2}} & \frac{c^{2} g \beta \rho}{a b-c^{2}} & -\frac{k_{d} b}{a b-c^{2}} & 0 \\ \frac{k_{s} c \beta}{a b-c^{2}} & -\frac{a c g \rho}{a b-c^{2}} & \frac{k_{d} c \beta}{a b-c^{2}} & 0\end{array}\right]$,

$B_{1}=B_{3}=\left[\begin{array}{c}0 \\ 0 \\ \frac{b}{a b-c^{2} \beta^{2}} \\ -\frac{c}{a b-c^{2} \beta^{2}}\end{array}\right], \quad B_{2}=B_{4}=\left[\begin{array}{c}0 \\ 0 \\ \frac{b}{a b-c^{2}} \\ -\frac{c}{a b-c^{2}}\end{array}\right], \quad B_{5}=B_{7}=\left[\begin{array}{c}0 \\ 0 \\ \frac{b}{a b-c^{2} \beta^{2}} \\ -\frac{c \beta}{a b-c^{2} \beta^{2}}\end{array}\right], \quad B_{6}=B_{8}=\left[\begin{array}{c}0 \\ 0 \\ \frac{b}{a b-c^{2}} \\ -\frac{c \beta}{a b-c^{2}}\end{array}\right]$, 


$$
\begin{aligned}
& F_{1}=F_{3}=\left[\begin{array}{cc}
0 & 0 \\
0 & 0 \\
\frac{b}{a b-c^{2} \beta^{2}} & -\frac{c}{a b-c^{2} \beta^{2}} \\
-\frac{c}{a b-c^{2} \beta^{2}} & \frac{a}{a b-c^{2} \beta^{2}}
\end{array}\right], F_{2}=F_{4}=\left[\begin{array}{cc}
0 & 0 \\
0 & 0 \\
\frac{b}{a b-c^{2}} & -\frac{c}{a b-c^{2}} \\
-\frac{c}{a b-c^{2}} & \frac{a}{a b-c^{2}}
\end{array}\right] \text {, } \\
& F_{5}=F_{7}=\left[\begin{array}{cc}
0 & 0 \\
0 & 0 \\
\frac{b}{a b-c^{2} \beta^{2}} & -\frac{c \beta}{a b-c^{2} \beta^{2}} \\
-\frac{c \beta}{a b-c^{2} \beta^{2}} & \frac{a}{a b-c^{2} \beta^{2}}
\end{array}\right], F_{6}=F_{8}=\left[\begin{array}{cc}
0 & 0 \\
0 & 0 \\
\frac{b}{a b-c^{2}} & -\frac{c \beta}{a b-c^{2}} \\
-\frac{c \beta}{a b-c^{2}} & \frac{a}{a b-c^{2}}
\end{array}\right], H_{a 1}=\left[\begin{array}{c}
0 \\
0 \\
\frac{b c \alpha^{2}}{a b-c^{2} \beta^{2}} \\
-\frac{c^{2} \alpha^{2}}{a b-c^{2} \beta^{2}}
\end{array}\right], \\
& H_{a 2}=\left[\begin{array}{c}
0 \\
0 \\
\frac{b c \alpha^{2}}{a b-c^{2}} \\
-\frac{c^{2} \alpha^{2}}{a b-c^{2}}
\end{array}\right], H_{a 3}=\left[\begin{array}{c}
0 \\
0 \\
\frac{b c \alpha^{2} \rho}{a b-c^{2} \beta^{2}} \\
-\frac{c^{2} \alpha^{2} \rho}{a b-c^{2} \beta^{2}}
\end{array}\right], H_{a 4}=\left[\begin{array}{c}
0 \\
0 \\
\frac{b c \alpha^{2} \rho}{a b-c^{2}} \\
-\frac{c^{2} \alpha^{2} \rho}{a b-c^{2}}
\end{array}\right], H_{a 5}=\left[\begin{array}{c}
0 \\
0 \\
\frac{b c \alpha^{2}}{a b-c^{2} \beta^{2}} \\
-\frac{c^{2} \alpha^{2} \beta}{a b-c^{2} \beta^{2}}
\end{array}\right] \\
& H_{a 6}=\left[\begin{array}{c}
0 \\
0 \\
\frac{b c \alpha^{2}}{a b-c^{2}} \\
-\frac{c^{2} \alpha^{2} \beta}{a b-c^{2}}
\end{array}\right], H_{a 7}=\left[\begin{array}{c}
0 \\
0 \\
\frac{b c \alpha^{2} \rho}{a b-c^{2} \beta^{2}} \\
-\frac{c^{2} \alpha^{2} \beta \rho}{a b-c^{2} \beta^{2}}
\end{array}\right], H_{a 7}=\left[\begin{array}{c}
0 \\
0 \\
\frac{b c \alpha^{2} \rho}{a b-c^{2}} \\
-\frac{c^{2} \alpha^{2} \beta \rho}{a b-c^{2}}
\end{array}\right] \text {. }
\end{aligned}
$$

For simulation purpose, the maximal angular velocity has been set as $\alpha=4 \pi \mathrm{rad} \cdot \mathrm{s}^{-1}$ and the maximal angular position as $\theta_{0}=\frac{\pi}{2}$. A convenient non-PDC SOFC (6) has been designed through theorem 2 and the Matlab LMI toolbox with the bounds of the membership functions sets as $\phi_{i}=-10$. The result is given by the following gain matrices for a minimal attenuation level $\lambda=0.8341$ :

$$
W_{1}^{5}=\left[\begin{array}{cc}
0.9667 & 0 \\
0 & 0.9588
\end{array}\right], W_{2}^{5}=\left[\begin{array}{cc}
0.9668 & 0 \\
0 & 0.9588
\end{array}\right], W_{3}^{5}=\left[\begin{array}{cc}
0.9663 & 0 \\
0 & 0.9588
\end{array}\right], W_{4}^{5}=\left[\begin{array}{cc}
0.9675 & 0 \\
0 & 0.9588
\end{array}\right] \text {, }
$$




$$
\begin{aligned}
& W_{5}^{5}=\left[\begin{array}{cc}
0.964 & 0 \\
0 & 0.9588
\end{array}\right], W_{6}^{5}=\left[\begin{array}{cc}
0.9639 & 0 \\
0 & 0.9588
\end{array}\right], W_{7}^{5}=\left[\begin{array}{cc}
0.9646 & 0 \\
0 & 0.9588
\end{array}\right], W_{8}^{5}=\left[\begin{array}{cc}
0.9638 & 0 \\
0 & 0.9588
\end{array}\right], \\
& L_{1}=\left[\begin{array}{ll}
0.2202 & -0.0009
\end{array}\right], L_{2}=\left[\begin{array}{ll}
0.2555 & -0.0021
\end{array}\right], L_{3}=\left[\begin{array}{ll}
0.2245 & -0.0001
\end{array}\right], L_{4}=\left[\begin{array}{ll}
0.2202 & -0.0007
\end{array}\right], \\
& L_{5}=\left[\begin{array}{ll}
0.1238 & 0.0022
\end{array}\right], L_{6}=\left[\begin{array}{ll}
0.0906 & 0.0033
\end{array}\right], L_{7}=\left[\begin{array}{ll}
0.1793 & 0.002
\end{array}\right] \text { and } L_{8}=\left[\begin{array}{ll}
0.1612 & 0.0011
\end{array}\right] .
\end{aligned}
$$

For simulation purpose, the external disturbances $\varphi_{1}(t)$ and $\varphi_{2}(t)$ has been respectively set as white noises with unit variance characterized by bounded energy added to steps such that $\varphi_{1} \approx 10$ for $t \in[1 s, 2 s]$ ( 0 elsewhere), and $\varphi_{2} \approx-10$ for $t \in[3 s, 3.5 s]$ ( 0 elsewhere). Simulations where performed with and without external disturbance to highlight the efficiency of the $H_{\infty}$ attenuation. Fig 6 and 7 show respectively the system's state, the control signal and the disturbance evolutions for the initial conditions $x(0)=\left[\begin{array}{llll}1 & \frac{\pi}{4} & 0 & 0\end{array}\right]^{T}$. It can be conclude that the synthesized robust non-PDC SOFC is correctly controlling the crane system in spite of the presence of external disturbances.

\section{Conclusion}

In this paper, the problem of robust static output feedback stabilization for continuous time uncertain and disturbed Takagi-Sugeno models has been considered. A non-PDC static output feedback control law has been proposed and its design has been involved through a fuzzy Lyapunov approach. Thanks to the descriptor redundancy, crossing terms have been avoided in the closed-loop dynamic formulation and so LMI conditions have been obtained without any assumptions on the output equation of the considered TS model. Then, a $H_{\infty}$ criterion has been employed to derive conditions which ensure a minimal attenuation level of external disturbances. It has been shown that the proposed SOFC based design lead to low conservatism results regarding to previous works. Moreover, it has been emphasis 
that, thank to the uncertain TS modeling, such approach is suitable for the design of a robust non-PDC static output feedback controller without the need of unmeasurable variables estimations. Finally, two academic examples has been considered to illustrate the efficiency the proposed fuzzy Lyapunov based SOFC design.

\section{Acknowledgements}

This work was supported by the French Ministry of Research and the "Région Champagne-Ardennes" within the CPER MOSYP. The authors would also like to thanks Miss Dolly Prane for her support within this study.

\section{References}

Assawinchaichote, W., Nguang, S.K., Shi, P., 2004. Output feedback control design for uncertain singularly perturbed systems: an LMI approach. Automatica 40 (12), 2147-2152.

Bernal, M., Guerra, T.M., 2010. Generalized non-quadratic stability of continuous-time Takagi-Sugeno models. IEEE Transactions on Fuzzy Systems 18 (4), 815-822.

Bouarar, T., Guelton, K., Mansouri, B., Manamanni, N., 2007. LMI Stability Conditions for TakagiSugeno Uncertain Descriptors. In: IEEE International Conference on Fuzzy Systems, FUZZ-IEEE 2007, London, UK, 23-26 July.

Bouarar, T., Guelton, K., Manamanni, N., 2008. Stabilization of uncertain Takagi-Sugeno descriptors: a fyzzy Lyapunov approach. In: 16th IEEE Mediterranean Conference on Control and Automation, MED 2008, Ajaccio, Corsica, France, pp. 1616-1621. 
Bouarar, T., Guelton, K., Manamanni, N., 2009. Static output feedback controller design for TakagiSugeno systems - A fuzzy Lyapunov LMI approach. In: 48th IEEE Conference on Decision and Control, CDC 2009, Shanghai, China, pp. 4150-4155.

Bouarar, T., Guelton, K., Manamanni, N., 2010. Robust fuzzy Lyapunov stabilization for uncertain and disturbed Takagi-Sugeno descriptor. ISA Transactions 49 (4), 447-461.

Chadli, M., Maquin, D., Ragot, J., 2002. Static output feedback for Takagi-Sugeno systems: an LMI approach. In: 10th IEEE Mediterranean Conference on Control and Automation, Lisbon, Portugal, pp.1035-1038.

Chang, X.H., Yang, G.H., 2010. Relaxed stabilization conditions for continuous-time Takagi-Sugeno fuzzy control systems. Information Sciences 180 (17), 3273-3287.

Chang, X.H., Yang, G.H., 2011. A descriptor representation approach to observer-based Hळ control synthesis for discrete-time fuzzy systems. Fuzzy Sets and Systems 185(1), 38-51.

Chadli, M., Guerra, T.M., 2012. LMI solutions for robust static output feedback control of TakagiSugeno fuzzy models. IEEE Transactions on Fuzzy Systems, in press, doi: 10.1109/TFUZZ.2012.2196048.

Chen, G., 2004. System analysis using redundancy of descriptor representation. In: IEEE International Symposium on Computer Aided Control Systems Design, Taipei, Taiwan, pp. 231-236.

Feng, G., 2006. A Survey on Analysis and Design of Model-Based Fuzzy Control Systems. IEEE Transactions on Fuzzy Systems 14 (5), 676- 697.

Guelton, K., Bouarar, T., Manamanni, N., 2008. Fuzzy Lyapunov LMI based output feedback stabilization of Takagi-Sugeno systems using descriptor redundancy. In: IEEE International Conference on Fuzzy Systems (IEEE World Congress on Computational Intelligence), FUZZ-IEEE 2008, Hong Kong, pp. 1212-1218. 
Guelton, K., Bouarar, T., Manamanni, N., 2009. Dynamic output feedback fuzzy Lyapunov stabilization of Takagi-Sugeno systems - a descriptor redundancy approach. Fuzzy Sets and Systems 160 (19), 2796-2811.

Guelton, K., Guerra, T.M., Bernal, M., Bouarar, T., Manamanni, N., 2010. Comments on fuzzy control systems design via fuzzy Lyapunov functions IEEE Transactions on Systems Man, Cybernetics. B 40 (3), 970-972.

Guerra, T.M., Bernal, M., Kruszewski, A., Afroun, M., 2007. A way to improve results for the stabilization of continuous-time fuzzy descriptor models. In: 46th IEEE Conference on Decision and Control, CDC 2007, New Orleans, USA, pp. 5960-5964.

Guerra, T.M., Bernal, M., Guelton, K., Labiod, S., 2012. Non-quadratic local stabilization for continuous-time Takagi-Sugeno models. Fuzzy Sets and Systems 201, 40-54.

Guerra, T.M., Kruszewski, A., Vermeiren, L., Tirmant, H., 2006. Conditions of output stabilization for nonlinear models in the Takagi-Sugeno's form. Fuzzy Sets and Systems 157 (9), 1248-1259.

Guerra, T.M., Vermeiren, L., 2004. LMI based relaxed nonquadratic stabilizations for nonlinear systems in the Takagi-Sugeno's form. Automatica 40 (5), 823-829.

Huang, D., Nguang, S.K., 2006. Robust Hœ static output feedback control of fuzzy systems: An ILMI approach. IEEE Transactions on Systems Man, Cybernetics. B 36 (1), 216-222.

Huang, D., Nguang, S.K., 2007. Static output feedback controller design for fuzzy systems: An ILMI approach. Information Sciences 177 (14), 3005-3015.

Johansson, M., Rantzer, A., Arzen, K.E., 1999. Piecewise quadratic stability of fuzzy systems. IEEE Transactions on Fuzzy Systems 7 (6), 713-722.

Lam, H., Leung, F., 2007. LMI-based stability and performance conditions for continuous-time nonlinear systems in Takagi-Sugeno's form. IEEE Transactions on Systems, Man, and Cybernetics B 37 (5), 1396-1406. 
Li, J., Wang, H.O., Niemann, D., Tanaka, K., 2000. Dynamic parallel distributed compensation for Takagi-Sugeno fuzzy systems: An LMI approach. Information Sciences 123 (3-4), 201-221.

Ma, X.J., Sun, Z.Q., He, Y.Y., 1998. Analysis and design of fuzzy controller and fuzzy observer. IEEE Transactions on Fuzzy Systems (6), 41-50.

Mansouri, B., Manamanni, N., Guelton, K., Kruszewski, A., Guerra, T.M., 2009. Output feedback LMI tracking control conditions with Hos criterion for uncertain and disturbed T-S models. Information Sciences $179(4), 446-457$.

Mozelli, L.A., Palhares, R.M., Avellar, G.S.C., 2009a. A systematic approach to improve multiple Lyapunov function stability and stabilization conditions for fuzzy systems. Information Sciences $179(8), 1149-1162$.

Mozelli, L.A., Palhares, R.M., Souza, F.O., Mendes, E.M.A.M., 2009b. Reducing conservativeness in recent stability conditions of TS fuzzy systems. Automatica 45 (6), 1580-1583.

Nguang, S.K., Shi, P., 2006. Robust Hळ output feedback control design for fuzzy dynamic systems with quadratic stability constraints: An LMI approach. Information Sciences 176 (15), 2161-2191.

Rhee, B.J., Won, S., 2006. A new Lyapunov function approach for a Takagi-Sugeno fuzzy control system design. Fuzzy Sets and Systems 157 (9), 1211-1228.

Sala, A., 2009. On the conservativeness of fuzzy and fuzzy-polynomial control of nonlinear systems. Annual Reviews in Control 33 (1), 48-58.

Sala, A., Guerra, T.M., Babuska, R., 2005. Perspectives of fuzzy systems and control. Fuzzy Sets and Systems $153(3), 432-444$.

Syrmos, V.L., Abdallah, C.T., Dorato, P., Grigoriadis, K., 1997. Static output feedback - A survey. Automatica 33 (2), 125-137.

Takagi, T., Sugeno, M., 1985. Fuzzy identification of systems and its applications to modeling and control. IEEE Transaction on Systems Man and Cybernetics 15 (1), 116-132. 
Tanaka, K., Hori, T., Wang, H.O., 2003. A multiple Lyapunov function approach to stabilization of fuzzy control systems. IEEE Transaction on Fuzzy Systems 11 (4), 582-589.

Tanaka, K., Ikeda, T., Wang, H.O., 1998. Fuzzy regulators and fuzzy observers: relaxed stability conditions and LMI-based designs. IEEE Transactions on Fuzzy Systems 6 (2), 1-16.

Tanaka, K., Ohtake, H., Wang, H.O., 2007. A Descriptor System Approach to Fuzzy Control System Design via Fuzzy Lyapunov Functions. IEEE Transactions on Fuzzy Systems 15 (3), 333- 341.

Tanaka, K., Wang, H.O., 2001. Fuzzy control systems design and analysis. A linear matrix inequality approach. Wiley: New York, USA.

Taniguchi, T., Tanaka, K., Wang, H.O., 2000. Fuzzy descriptor systems and nonlinear model following control. IEEE Transactions on Fuzzy Systems 8 (4), 442-452.

Tognetti, E.S., Oliveira, R.C.L.F., Peres, P.L.D., 2012. Reduced-order dynamic output feedback control of continuous-time T-S fuzzy systems. Fuzzy Sets and Systems 207, 27-44.

Tuan, H.D., Apkarian, P., Narikiyo, T., Yamamoto, Y., 2001. Parametrized linear matrix inequality techniques in fuzzy control design. IEEE Transactions on Fuzzy Systems 9 (2), 324-332.

Wang, H.O., Tanaka, K., Griffin, M.F., 1996. An approach to fuzzy control of nonlinear systems: stability and the design issues. IEEE Transactions on Fuzzy Systems 4 (1), 14-23.

Xiaodong, L., Qingling, Z., 2003. New approaches to Hœ controller design based on fuzzy observers for fuzzy T-S systems via LMI. Automatica 39 (9), 1571-1582.

Yoneyama, J., Nishikawa, M., Katayama, H., Ichikawa, A., 2000. Output stabilization of TakagiSugeno fuzzy systems. Fuzzy Sets and Systems 111 (2), 253-266.

Yoneyama, J., Nishikawa, M., Katayama, H., Ichikawa, A., 2001. Design of output feedback controllers for Takagi-Sugeno fuzzy systems. Fuzzy Sets and Systems 121 (1), 127-148.

Zerar, M., Guelton, K., Manamanni, N., 2008. Linear fractional transformation based H-infinity output stabilization for Takagi-Sugeno fuzzy models. Mediterranean Journal of Measurement and Control $4(3), 111-121$. 
Zhou, K., Doyle, J.C., 1996. Robust optimal control. Prentice-Hall, New Jersey, USA.

Zhou, K., Khargonekar, P.P., 1988. Robust stabilization of linear systems with norm-bounded timevarying uncertainty. Systems Control Letters 10, 17-20. 
Figures and tables :

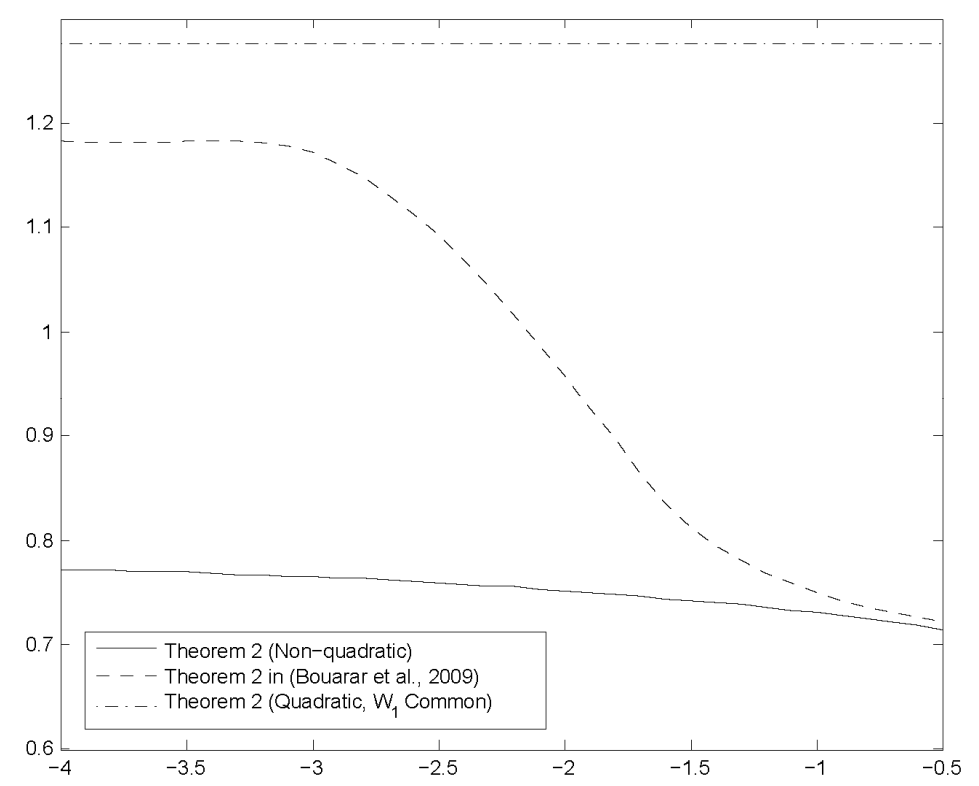

Fig. 1: Attenuation level $\lambda$ for several values of $\phi_{1}$ (example 1).
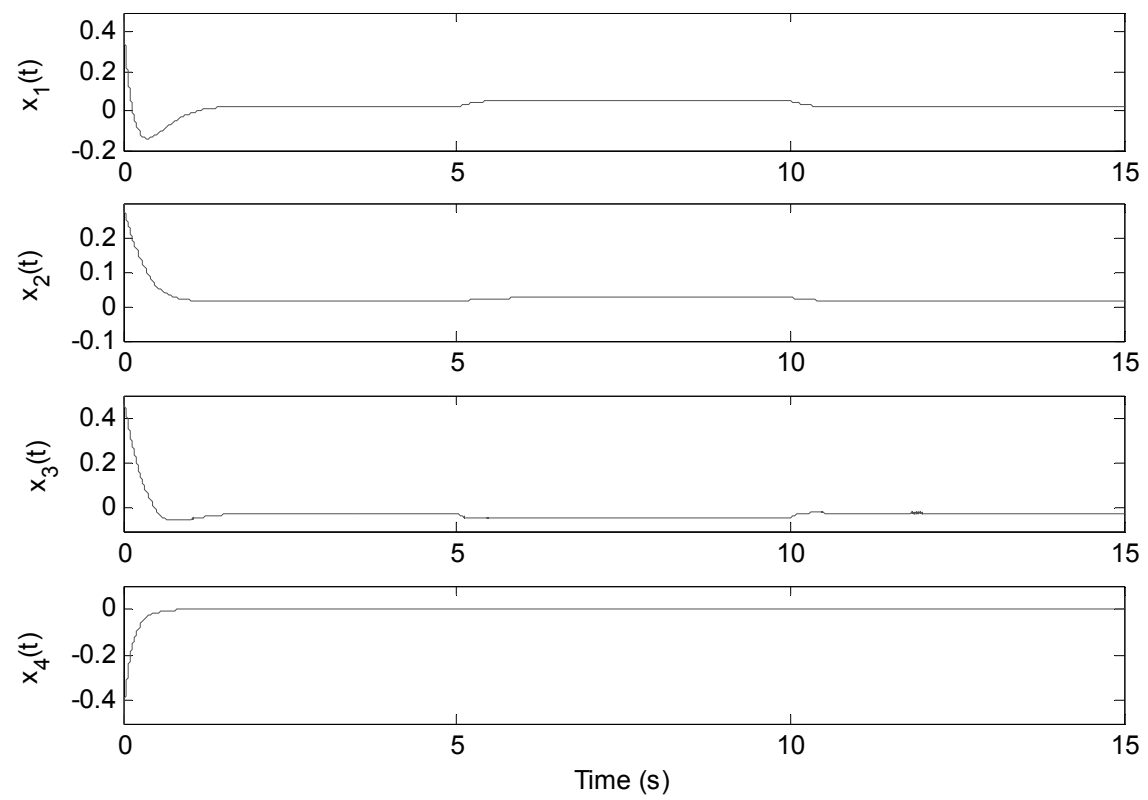

Fig. 2: Time responses of the T-S system's states with external disturbances (example 2) 

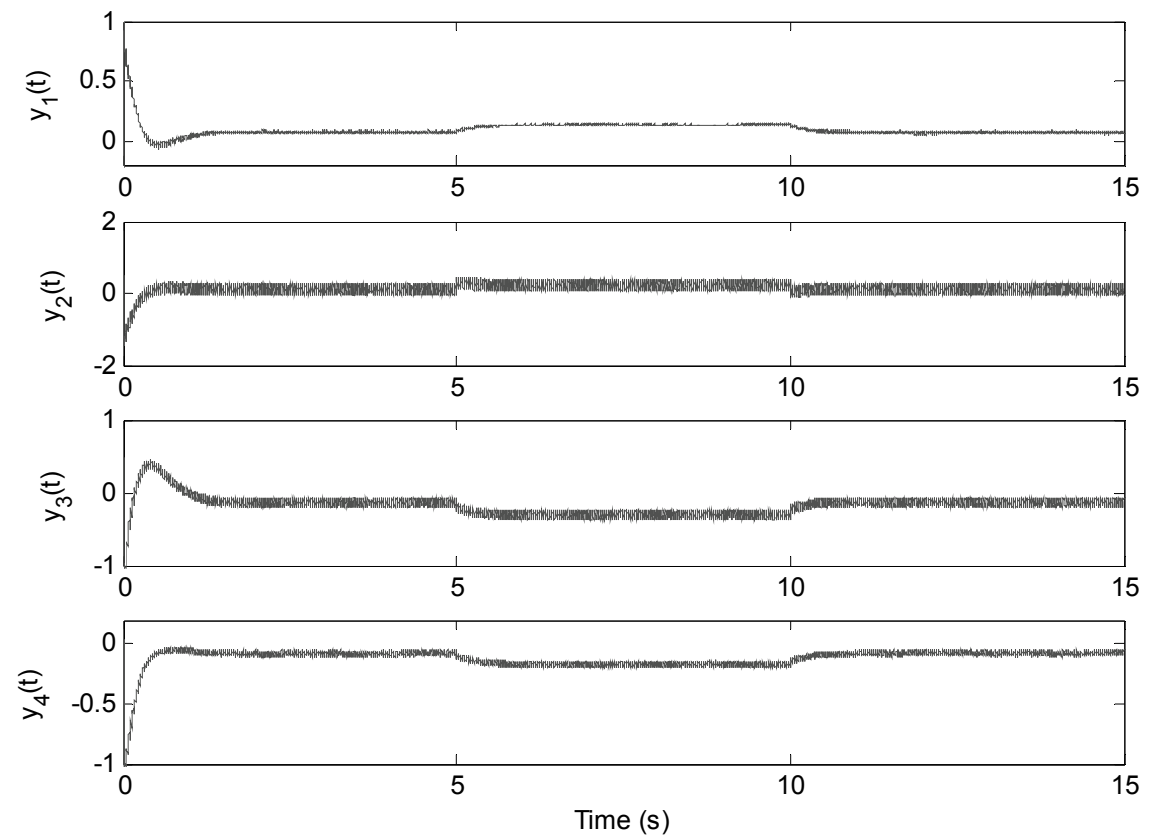

Fig. 3: Evolution of the outputs of the T-S systems with external disturbances (example 2)
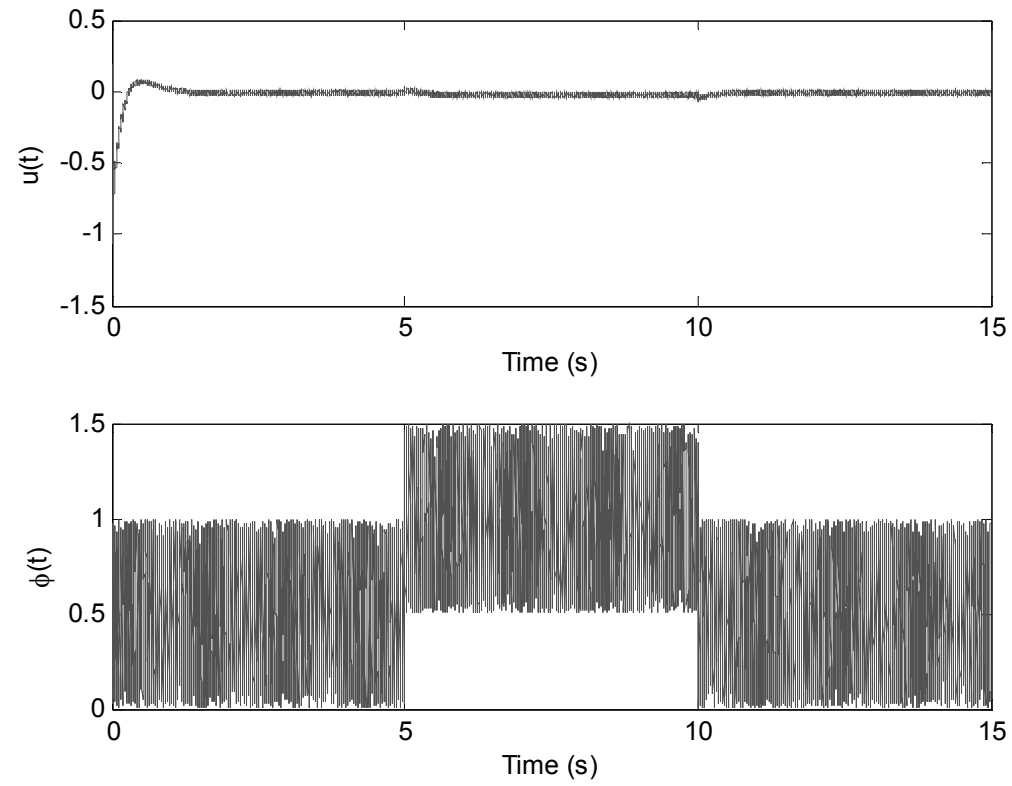

Fig. 4: Evolution of the control signal and external disturbances (example 2). 


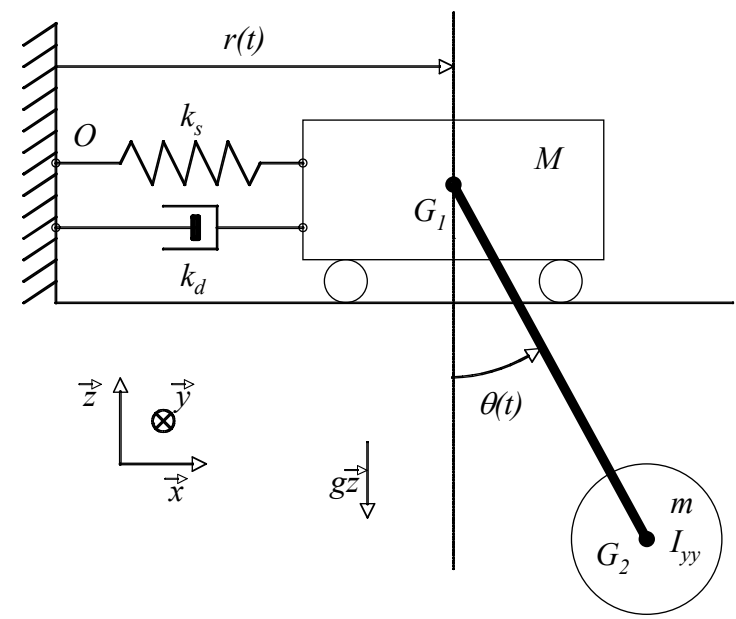

Fig.5. Crane system (example3).

Table 1. Model parameters of the crane system (example 3).

\begin{tabular}{|l|l|l|}
\hline Parameters & Designation & Value \\
\hline$m$ & Pendulum mass & $10(\mathrm{~kg})$ \\
\hline$M$ & Cart mass & $3(\mathrm{~kg})$ \\
\hline$k_{s}$ & Spring stiffness & 10 \\
\hline$k_{d}$ & Damping coefficient & 10 \\
\hline$L$ & Length of the pendulum & $0.5(\mathrm{~m})$ \\
\hline$I_{y y}$ & Inertia of the pendulum around the $\vec{y}$ axis & $0.3\left(\mathrm{~kg} / \mathrm{m}^{2}\right)$ \\
\hline$g$ & Gravity acceleration & $9.81\left(\mathrm{~m} / \mathrm{s}^{2}\right)$ \\
\hline$r(t)$ & Position of the cart along the $\vec{x}$ axis & $-(\mathrm{m})$ \\
\hline$\theta(t)$ & Pendulum angular position & $-(\mathrm{rad})$ \\
\hline$u(t)$ & Input signal (Force on the cart within $\vec{x})$ & $-(\mathrm{Nm})$ \\
\hline
\end{tabular}



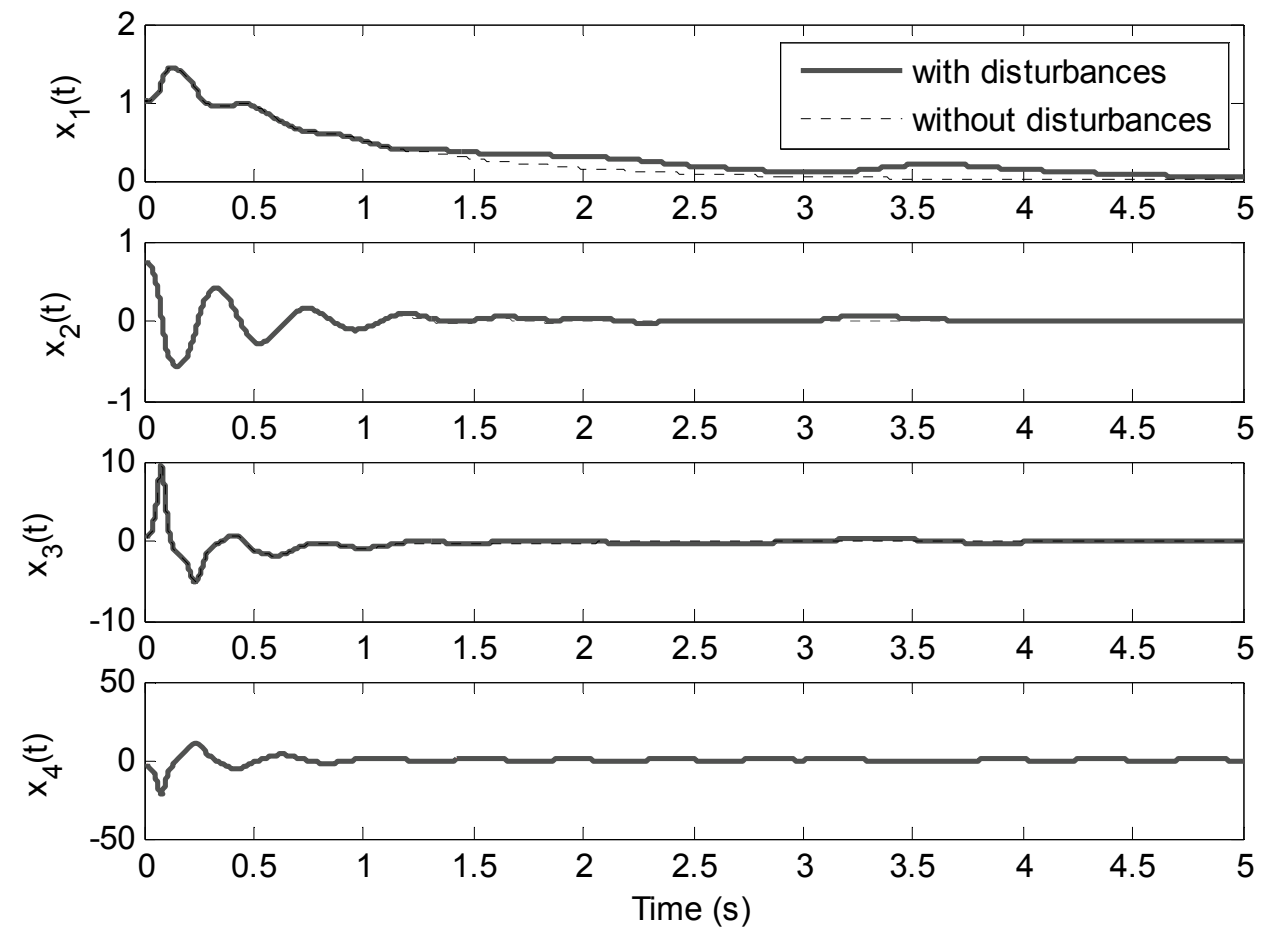

Fig. 6. Evolution of the states of the crane system (example 3).
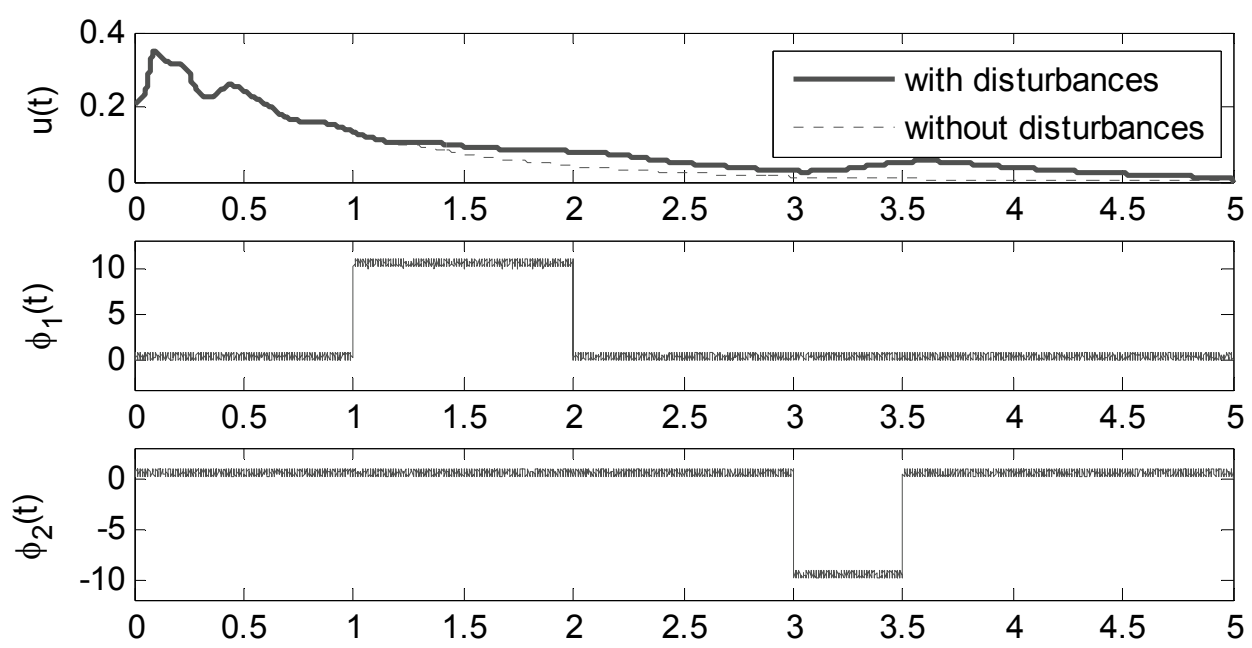

Fig. 7. Evolution of the control signal and external disturbances (example 3). 